\title{
Re-design of an Inbound Planning Interface for Air Traffic Control
}

\author{
R. E. Klomp*, M. Mulder ${ }^{\dagger}$, M. M. van Paassen ${ }^{\ddagger}$ \\ Delft University of Technology, Faculty of Aerospace Engineering \\ Delft, The Netherlands \\ M. I. Roerdink ${ }^{\S}$ \\ Air Traffic Control The Netherlands (LVNL) \\ Schiphol-Oost, The Netherlands
}

\begin{abstract}
In the coming decades, the task of an air traffic controller is expected to shift to one of strategic, trajectorybased air traffic management. This form of air traffic control is no longer possible without the help of automated support tools. In previous research, it has been shown that the time-space diagram, combined with a conventional plan view display is a good candidate for supporting an air traffic controller with the inbound planning task in the future situation. However, in this initial study, the vertical plane was not yet fully included. Secondly, during an initial validation experiment, creating and maintaining a 'mental picture' of the traffic was reported to be a difficult task. These findings lead to the re-design of the interface in the current research, which focuses on implementing the vertical plane and improving the integration of information across the successive displays. An experiment has been performed with a PC-based simulation which validates that the enhanced interface can be used to manage the air traffic safely and efficiently. Secondly, it has been shown that the ability to manipulate the speed of an aircraft in the adjacent sector can significantly increase situation awareness and reduce controller workload.
\end{abstract}

\section{Acronyms}

\begin{tabular}{|c|c|c|c|}
\hline 4DT & Four-dimensional trajectory & HMI & Human-Machine Interface \\
\hline ACC & Area Control Center & IAF & Initial Approach Fix \\
\hline ACID & Aircraft Identification & IAS & Indicated Airspeed \\
\hline ANOVA & Analysis of Variance & LVNL & Luchtverkeersleiding Nederland \\
\hline APP & Approach Control (Center) & & (ATC The Netherlands) \\
\hline ATC & Air Traffic Control & PVD & Plan View Display \\
\hline ATCo & Air Traffic Controller & ROD & Rate of Descent \\
\hline ATM & Air Traffic Management & SA & Situation Awareness \\
\hline CAS & Calibrated Airspeed & TAS & True Airspeed \\
\hline CTA & Controlled Terminal Area & TLX & Task Load Index \\
\hline DMI & Direct Manipulation Interface & TMA & Terminal Maneuvering Area \\
\hline DUT & Delft University of Technology & TSD & Time-Space Diagram \\
\hline EID & Ecological Interface Design & TSVD & Time-Space and Vertical Display \\
\hline FMS & Flight Management System & VSD & Vertical Situation Diagram \\
\hline
\end{tabular}

${ }^{*}$ MSc. student, Control and Simulation Division, Faculty of Aerospace Engineering, Delft University of Technology, Kluyverweg 1, 2629 HS Delft, The Netherlands. E-mail: rolfklomp@hotmail.com

${ }^{\dagger}$ Professor, Control and Simulation Division, Faculty of Aerospace Engineering, Delft University of Technology, Kluyverweg 1, 2629 HS Delft, The Netherlands. Senior member AIAA. E-mail: m.mulder@tudelft.nl

${ }^{\ddagger}$ Associate Professor, Control and Simulation Division, Faculty of Aerospace Engineering, Delft University of Technology, Kluyverweg 1, 2629 HS Delft, The Netherlands. Member AIAA. E-mail: m.m.vanpaassen@ tudelft.nl

${ }^{\S}$ Human Factors Expert, Air Traffic Control The Netherlands (LVNL), Stationsplein ZuidWest 1001, 1117 CV Schiphol-Oost, The Netherlands. E-mail: m.roerdink@lvnl.nl 


\section{Introduction}

$\mathrm{C}$ URRENTLY, air traffic controllers (ATCos) perform a sector-based tactical form of control. They are responsible for planning and managing traffic within their assigned airspace, often with little help from automated tools. ${ }^{1,2}$ In the coming decades, the task of an air traffic controller is predicted to undergo a large transformation. The pull for transformation comes from the increasing demands which are placed on the air traffic management (ATM)-system..$^{3-5}$ A push is provided by technological advances on the air- and ground side of the ATM-system, which make a new form of air traffic control (ATC) possible. ${ }^{6,7}$

The proposed transformation of the ATM-system itself is mainly focused on increasing capacity, safety and efficiency, enabled by an increased amount of data exchange between the ground- (control centers, airports) and air (aircraft) segment. ${ }^{8-10}$ For this purpose, the four-dimensional trajectory (4DT) is introduced, which specifies an aircraft's planned trajectory in three spatial dimensions and in time. In a mature state, at all times, for all controlled aircraft, the 4DTs are envisioned to be available to both the control centers and the aircrew involved. ${ }^{6}$ Both the ATCo and aircrew will be able to negotiate changes to the plan via data-link, for instance to avoid bad weather, to assure separation, or to manage arriving and departing traffic. As a consequence, the task of an ATCo is expected to shift to one of strategic four-dimensional trajectory-based control. ${ }^{6,10,11}$ In this form of ATC, time is added as an explicit control variable, which will make the planning more flexible and accurate. However, due to the extra level of abstraction, this type of ATC is no longer possible without the support of automated tools.

In previous research, ${ }^{12}$ a human-machine interface (HMI) has been developed to visualize these 4DT's and support the air traffic controller in planning and managing the air traffic in this future situation. One of the main considerations during the design of the interface was to keep the air traffic controller fully in the loop of the decision making process and to let the human continue to be the key decision maker (human-centered automation ${ }^{13}$ ). The role of an Area Control Center (ACC)-ATCo was taken as a focal point, focusing on managing inbound streams of traffic through their designated airspace into the terminal maneuvering area (TMA) of an airport. Whereas the planned times along an aircraft 4DT are discretely displayed for a certain metering fix in other experimental interfaces, ${ }^{9}, 14-16$ the basis of the new HMI was formed by a dynamic time-space diagram (TSD). Following the principles of Ecological Interface Design (EID) ${ }^{17}$ the relevant constraints to the task of the ATCo were visualized on the TSD, forming a foundation on which the ATCo could base their decisions. With the HMI, an initial validation experiment has been performed, which showed that this type of interface indeed provides an ATCo the means to safely and efficiently manage air traffic. However, the experiment also revealed certain areas which are in need of further research.

The focus of the current research lies on enhancing the interface, following the recommendations made in the previous research. First, the TSD has been combined with a vertical situation diagram (VSD), which is used for visualizing additional constraints in the vertical plane, and to offer direct manipulation of the vertical profile. Secondly, the options to manipulate the 4DT of an aircraft have been expanded, providing the controller with more freedom in planning the trajectories. And finally, two new tools have been developed to aid the ATCo with interpreting the information shown on the TSD and VSD, and relating it to the spatial traffic situation on the plan view display (PVD). These are the visualization of conflicts, and the option to dynamically project the future traffic situation on the PVD. The current research resulted in a re-design of the interface, with which an experimental validation has been performed.

This paper describes the re-design and validation of the interface, and is structured as follows. In Section II, the hypothesized future situation for ACC is discussed, serving as a baseline scenario for which the interface has been designed. Section III gives a brief overview of the previous research, from which the findings and recommendations form the starting point for the re-design. In Section IV the enhancements made to the interface, and its implementation in a PC-based simulation, will be discussed in more detail. The validation experiment and the feedback and results which were obtained are described in Sections V and VI, respectively. The results are discussed in Section VII and the paper ends with conclusions and recommendations for future research.

\section{Future inbound traffic management}

In this section, the hypothesized future situation of air traffic management for an ACC-controller will be discussed, focused on managing inbound traffic. Here, the architecture of the ATM-system will make heavy use of time-based operations. The language in which the aircrew and ATCos communicate will be one of four-dimensional trajectories, any negotiation or renegotiation of a previously agreed trajectory will always result in a new 4DT. ${ }^{71}$ The amount of data required to make this possible implies that this type of ATC cannot be conducted by voice alone. Also, instructions which require immediate compliance by the pilot are not easily adapted to the amount of time which is required to enter them in the aircrafts flight management system (FMS) manually. ${ }^{6}$ Therefore, direct communication with an 
aircrafts FMS through data-link will likely come to play an essential part in the future task of an ATCo.

The assumption is made that traffic will be able to execute operator preferred trajectories in the en-route phase of flight, with little or no intervention from air traffic control. As a result, inbound traffic will enter the ACC sector from various directions, with various speeds and at various altitudes. Secondly, the assumption is made that inside the terminal maneuvering area (TMA) of an airport, three-dimensional fixed routes will be implemented. The main goal of this is to make (time-based) continuous descent approaches (CDAs) possible, ${ }^{18,19}$ reducing noise, and avoiding the spread of noise to densely populated areas. As a consequence, the task of inbound traffic management by an ACC-ATCo will focus on the trajectory-based merging of different streams of traffic, and on delivering the traffic to the entry points (IAFs) of the TMA at a precise time.

\section{A. Merging by Area Control}

Because the TMA of an airport usually has more entry points than there are landing runways in use, the various traffic streams have to be merged inside the TMA (Figure 1). When fixed routes are implemented inside the TMA, only small changes can be made to the final arrival time of an aircraft at the runway once it has physically entered the terminal sector. Consequently, in order to create a workable situation for the approach (APP) controller, the ACC-ATCo will have to precisely plan and execute the arrival times of the aircraft at the fix. Secondly, to achieve an efficient stream of traffic and a high landing capacity, the sequence of aircraft and wake-vortex spacing also has to be taken into account by the ACC-ACTo before the traffic enters the TMA.

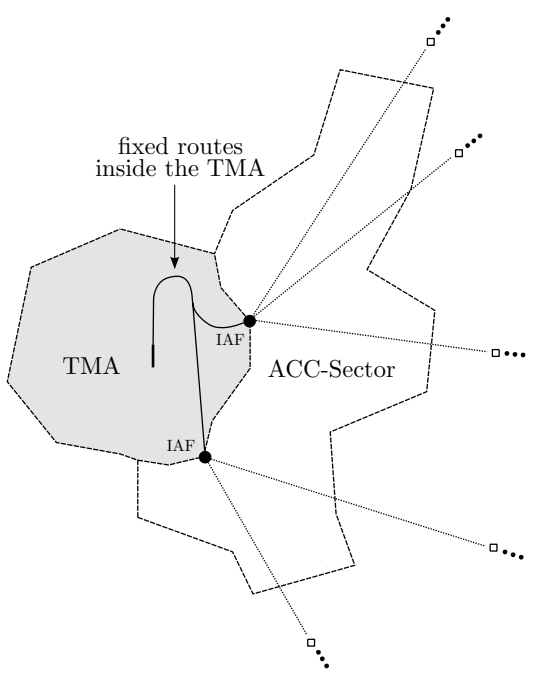

Figure 1: Three-dimensional fixed routes inside the TMA, starting at the Initial Approach Fixes. Outside the TMA, aircraft arrive from all directions and are merged by ACC.

\section{B. Multi-sector coordination: speed requests to the adjacent sector}

In most cases, Western European ATC sectors are relatively small, and the descent of an inbound aircraft often spreads though several sectors. If the quantity of inbound traffic from adjacent sectors temporarily exceeds the amount of traffic which can safely enter the TMA, aircraft will have to be delayed in the ACC sector. Delaying aircraft is often performed by means of path stretching (detour or holding pattern). However, a more efficient way in which to cope with these delays would be to already slow down aircraft in the adjacent sector, such that path stretching can be avoided. This could reduce ATCo workload, increase the efficiency of the overall traffic flow, and is often more efficient in terms of fuel consumption. In the experimental validation of the interface described in the following section, the option to create an extra margin in arrival times by this type of multi-sector coordination was found to be widely used and appreciated. However, the effect of issuing speed requests to the adjacent sector on the controller workload, Situation Awareness (SA), and to the overall efficiency of the traffic remained to be investigated. Therefore, this was addressed in the experimental validation which has been performed in the current research. 


\section{Previous research}

In our previous research, ${ }^{12}$ an interface has been developed for supporting the ACC-ATCo in performing this trajectory-based form of inbound air traffic control. The design of the interface followed the principles of Ecological Interface Design (EID), which focuses on how the environment, or ecology, imposes constraints on the work of a human operator. ${ }^{17}$ In order to let the (human) ATCo remain to be the key decision maker, the design was focused on graphically presenting the relevant constraints for the ATC task on the interface. Consequently, this presentation formed the foundation on which the ATCo could base their decisions. Secondly, since multi-sector coordination was found to be important for the efficiency of the traffic flow and to prevent path stretching, support for multi-sector coordination was added by visualizing whether a speed request to the adjacent sector is something that is useful and feasible.

\section{A. Basics of the HMI}

The planning interface was formed by a time-space diagram, which displays the planned times versus the along-track distance of an aircrafts 4DT. The identified constraints for the inbound planning task were then graphically translated onto the TSD, providing the ATCo with the necessary information to control the traffic safely and efficiently. Finally, the interface was constructed and evaluated in a PC-based simulation program. Figure 2 shows a detailed sketch of the interface, when a single aircraft is selected. As the HMI was designed to be used alongside the conventional plan view display, the PVD is also shown in the figure.

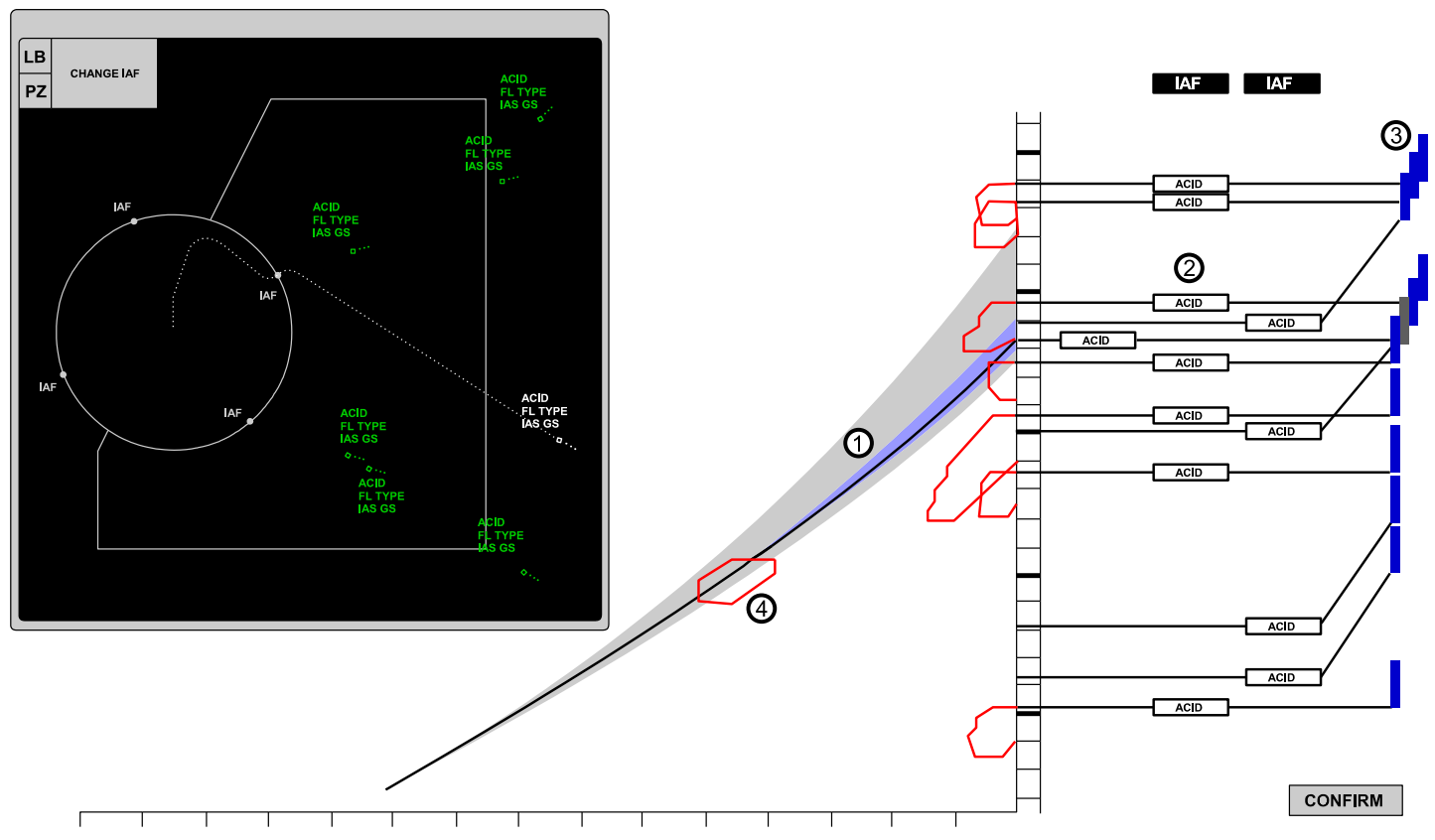

Figure 2: Detailed sketch of the interface used for evaluation in the previous research

The main elements of the interface, as shown in Figure 2, are:

1. Speed constraints: Speed constraints are visualized as 'triangles' on the TSD, indicating the feasible range of time-space lines (bounded by the aircrafts minimum and maximum speed) along the present trajectory. The outer triangle indicates the feasible range of time-space lines when a change to speed is issued in the adjacent sector. The inner triangle indicates the feasible range when a change to the aircrafts speed is made inside the own sector. At the time axis of the TSD, these constraints visualize the margin of arrival times at which the aircraft can enter the TMA by a change in speed alone. The currently selected time-space line of the aircraft is highlighted in black.

2. Traffic sequence: Labels containing the aircraft identification codes (ACID) of the inbound traffic are located to the right of the TSD, and are placed at the time at which the aircraft is planned to arrive at the fix. There are three columns. In the left column the ACID of the selected aircraft is shown. The labels of the other traffic are 
located in the middle and right columns, beneath their planned fix. By direct manipulation, a different arrival time at the fix can be selected by dragging the ACID of the selected aircraft along the time axis of the TSD.

3. Wake-vortex separation: The blue bars indicate the aircrafts time slot at the runway. The length of the blue bars relates to the required wake-vortex (time-based) separation between aircraft at the runway. The placement of the bars with respect to the aircrafts arrival time is dependent on the approach fix, and accounts for the difference in length of the two fixed routes inside the TMA. When the blue bars are neatly 'stacked' and do not overlap, this indicates a safe and efficient merging and planning inside the TMA.

4. Separation constraints: The forbidden zones on the TSD visualize regions in the time-space domain, through which the time-space line of an aircraft may not pass; there will be a loss of separation. The location along the horizontal axis indicates the along-track section of the trajectory which is occupied by conflicting traffic. The location along the vertical axis indicates the time of occupation.

\section{B. Initial validation experiment}

An (initial) experimental validation has been performed with the above displayed interface to find out two things:

- Can the interface actually be used to manage inbound traffic safely and efficiently?, and

- What should be the focus of further development?

Ten test subjects participated in the experiment; five of them certified air traffic controllers, the other five had relevant working experience in the ATC domain. The subjects were given four different traffic scenarios to solve, with increasing difficulty (higher aircraft count and bunching). They were asked to plan the trajectories of the inbound aircraft without loss of separation in the own sector, and to merge the traffic streams inside the TMA (stacking the blue bars). After the experiment, the subjects filled out a subjective questionnaire concerning the use and their overall appreciation of the interface.

\section{Conclusions and recommendations}

From the feedback obtained by the questionnaires, and the comments which were made by the test subjects during the validation, a number of conclusions and recommendations were drawn.

The new interface indeed provides the means for an ATCo to manage inbound traffic safely and efficiently, without loss of separation. Almost all test subjects agreed that the direct manipulation of the arrival time at the fix worked pleasantly. Secondly, the importance of multi-sector coordination was underlined by the fact that all ATCos liked to use the option of issuing speed requests to the adjacent sector.

However, the results also revealed areas of the HMI which were in need of further research. The fact that the vertical dimension could not be controlled was seen as a deficiency by the ATCos, who often use the vertical plane to separate aircraft in the current operation. Also, there was no clear relationship between the generation of the forbidden zones in the TSD and the vertical profile of the aircraft. Secondly, the level of situation awareness was reported to be poor. Combining the information displayed on the TSD and PVD to create a complete mental picture was found to be difficult for almost all test subjects. The planning interface was often used as a primary tool for controlling the traffic, neglecting the PVD completely and fully trusting on the information displayed in the TSD. Therefore, the following recommendations have been made:

- The manipulation of the vertical profile, and its relation with the TSD should be integrated in the interface to make it more complete,

- Coordination between adjacent sectors should remain part of the future development, and

- Adding sequence numbers to aircraft and displaying conflicts on the PVD could improve the integration of information. 


\section{The re-designed interface}

For the current research, the overall goal of developing an interface to support the ACC-ATCo in a future trajectorybased form of ATC remains the same. The previously developed interface forms the starting point to which a number of enhancements have been made. From the conclusions and recommendations which followed from the initial validation experiment, three main areas of focus have been derived. These are:

- Introducing the vertical plane,

- Enhancing the control options for manipulating a 4DT, including the vertical profile as a control variable, and

- Improving the integration of information between the planning interface and plan view display to increase situation awareness.

\section{A. Introduction of the vertical plane}

The plan view display and time-space diagram are used to visualize the horizontal path and planned times of a 4DT, but do not give any direct or continuous information about the planned vertical path. However, when considering the constraints posed to the task of the ATCo, most have a direct relationship with the vertical plane. Conflicts between aircraft can be resolved by vertical separation, and the vertical profile has a direct influence on the feasible speeds and the geometry of the 4DT. The combination of a PVD and TSD alone, therefore, only provides a partial representation of the environment and boundaries within which the ATCo has the freedom to control in current operations. As a starting point, a vertical situation diagram (VSD) has been added to make the interface more complete.

\section{Vertical Situation Diagram (VSD)}

To support the ATCo (or any controller of a complex system) with creating a solid and correct 'mental picture' of the controlled system, the basic perception of important and relevant information is crucial. ${ }^{20}$ Therefore, a clear and continuous representation of the vertical plane is a necessary addition to the interface. When an aircraft is capable of following a precise 4DT, for many points along its trajectory, a spatial location, altitude and time are known at which the aircraft is planned to pass that point. In the previous research it was shown how a TSD can be created by connecting the times at these consecutive points. Similar to the generation of a TSD, by connecting the consecutive altitudes, a VSD can be constructed. As the altitudes and times are obtained from the same along-track distances, these two diagrams can be combined.

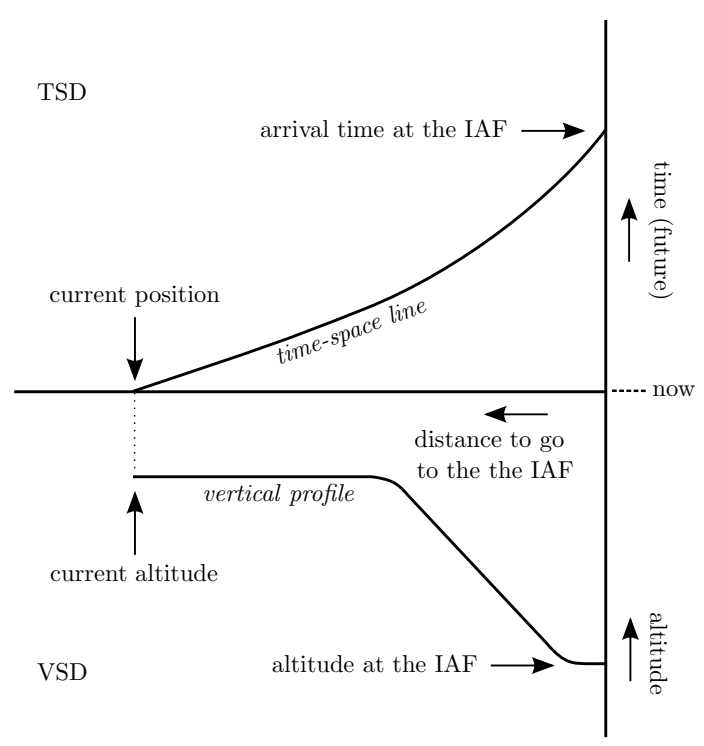

Figure 3: A TSD combined with a VSD of an aircraft flying to an IAF 
An example of the combination of a time-space diagram and a vertical situation diagram is given in Figure 3 , for an inbound aircraft which is en-route to an IAF. The top graph is the TSD indicating planned time versus distance to go to the IAF. The bottom graph is the VSD indicating planned altitude versus the same distance to go. The current distance of the aircraft from the fix is indicated at the intersection of its time-space line with the horizontal axis. The current altitude of the aircraft is indicated directly below the current position. From the TSD, the arrival time at the IAF follows from the time at the intersection of the time-space line with the vertical axis. In the VSD, the aircrafts vertical profile is shown along its trajectory. As time progresses, the vertical (time) axis of the TSD will shift downwards. The intersection of the time-space line with the horizontal axis, together with current location along the vertical profile, will then shift to the right (towards the IAF).

As the interface is now expanded with a continuous visualization of the vertical plane, the VSD will serve as a basis onto which the additional vertical constraints will be mapped.

\section{Vertical separation constraints}

The most important task of any ATCo is to keep the traffic conflict free at all times. In civil ATC sectors, the required minimum separation between a pair of aircraft is typically defined as $5 \mathrm{NM}$ horizontally and 1,000ft vertically. These will be referred to as the horizontal and vertical separation criterion respectively. Subsequently, a conflict occurs when both criteria are violated at the same time. A cylindrical shaped zone can be defined around each aircraft, called a protected zone, in which other aircraft may never enter.

In the previous research it was shown how these protected zones can be translated to forbidden zones on the TSD. With the addition of the VSD, forbidden zones can now also be visualized in the vertical plane. Figure 4 shows an example of how such a zone can be generated in the VSD of aircraft $A$ due to aircraft $B$. In this figure, the separation circles around both aircraft indicate a radius of $2.5 \mathrm{NM}$. As a consequence, the horizontal separation is violated when the circles overlap. Aircraft $A$ and $B$, both flying at a constant altitude, will perpendicularly cross each other's trajectory, resulting in a violation of the horizontal separation criterion at a certain point in time. Note that in the final VSD the vertical profile of aircraft $A$ is also shown, however, it has been omitted from Figure 4.

Figure 4.a shows how the horizontal trajectory of aircraft $A$ can be projected onto the horizontal axis of its VSD. At a certain time $t_{1}$, at distance $d_{1}$ along the trajectory of aircraft $A$, the horizontal separation criterion between both aircraft will be violated as shown in Figure 4.b. At a certain time $t_{2}$, at distance $d_{2}$, both aircraft will have passed each other, restoring horizontal separation, as shown in figure 4.c. Figure 4.d shows the generated forbidden zone in the VSD of aircraft $A$ due to the path of aircraft $B$. The forbidden zone is vertically located at the altitude of aircraft $B$, and has a height of positive and negative 1,000ft. The horizontal location follows from the section along the trajectory of aircraft $A$ where the separation circles of both aircraft overlap. The shape and size of the forbidden zones in the VSD are dependent on the geometry and the speeds of the 4DT's of both aircraft.
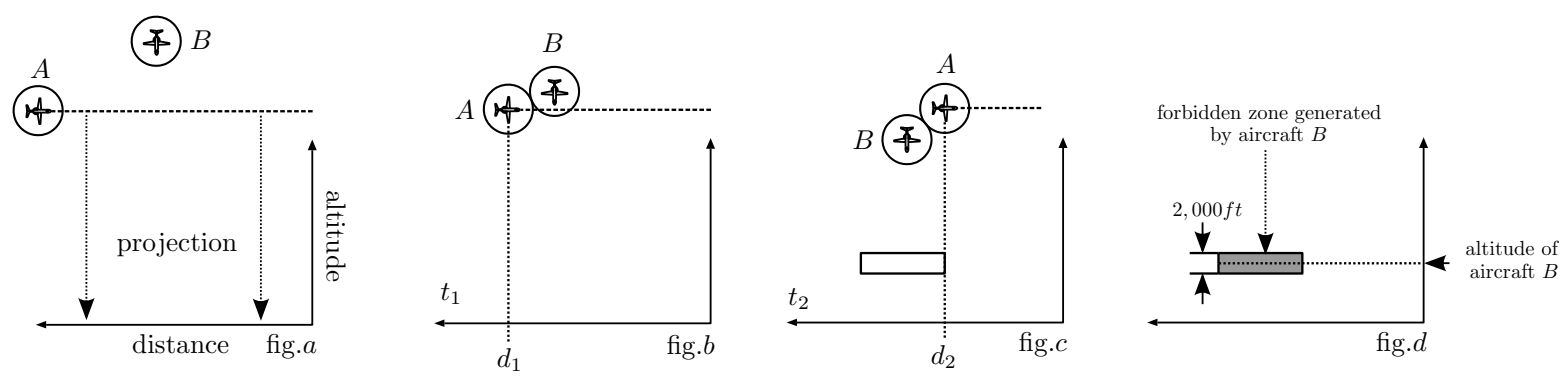

Figure 4: Example of the generation of a forbidden zone in the VSD of aircraft $A$, caused by aircraft $B$ perpendicularly crossing its trajectory at a constant altitude 
Sketches of typical forbidden zones created by conflicts in the vertical plane are shown in Figure 5. Here, the VSD belongs to aircraft $A$, and the forbidden zones are caused by aircraft $B$. The solid line represents the vertical profile of aircraft $A$, and the dotted line indicates the contour of the forbidden zone. When the vertical profile passes through a forbidden zone, aircraft $A$ will be located inside the protected zone of aircraft $B$ at a certain point along the trajectory; i.e., there will be a loss of separation.
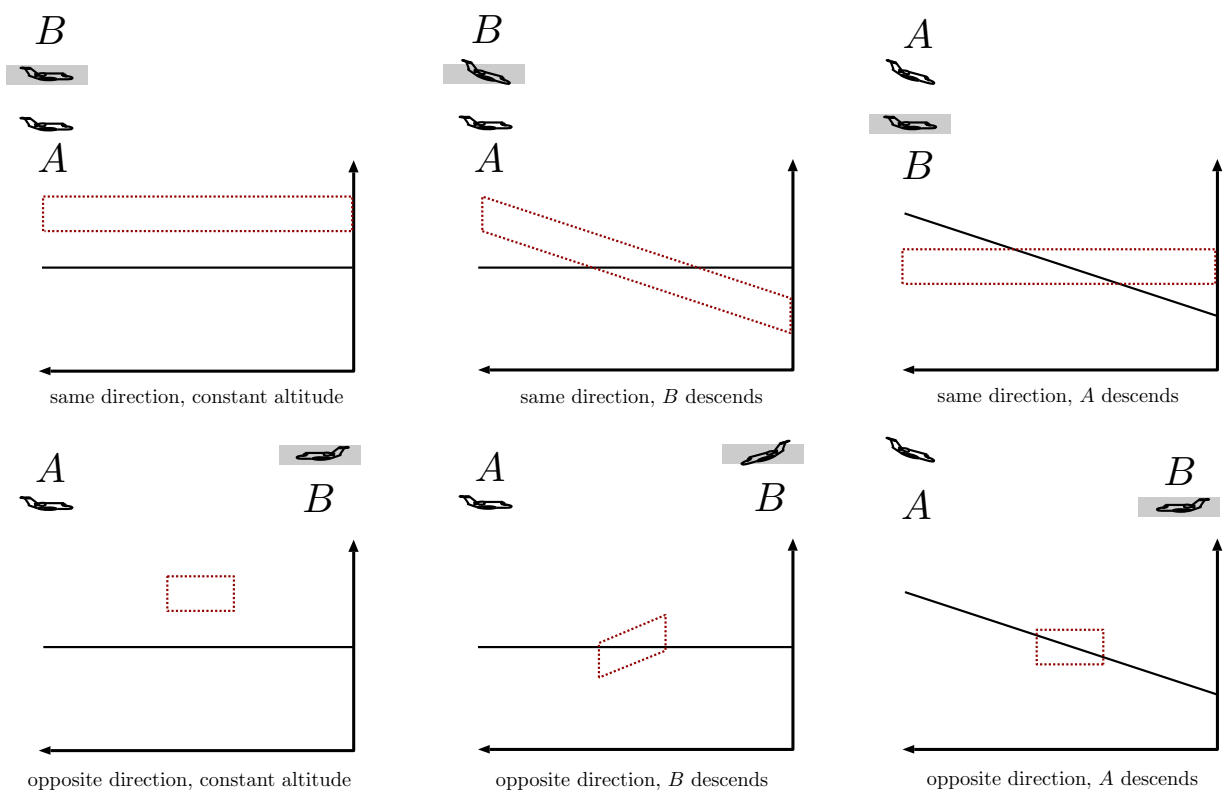

Figure 5: Sketches of typical forbidden zones in the VSD of aircraft $A$, due to aircraft $B$, for situations in the vertical plane

\section{Relationship between the forbidden zones in the TSD and VSD}

As forbidden zones are now visualized in both the TSD and VSD, it is important to clarify their mutual relationship. For this purpose, the zones will be classified in two categories; potential- and real conflicts. Potential conflicts are forbidden zones through which an aircrafts time-space line and vertical profile do not pass. These have a cautionary function, and can be useful for the ATCo when manipulating a 4DT.

Per definition, the cautionary zones visualized on the TSD and VSD cannot originate from the same cause. For zones in the VSD: the vertical separation implies that the conflicting aircraft will never actually intersect with the trajectory of the observed aircraft. Similarly for the TSD: the time-based separation implies that the horizontal separation criterion between the two aircraft will never actually be violated.

On the other hand, real conflicts will always result in a mutual pair of forbidden zones. A real conflict implies that the observed aircraft will be located inside the protected zone of a conflicting aircraft along a certain section of its trajectory. Consequently, both the horizontal and vertical separation criterion will then be violated. Relating this to the forbidden zones shown on both diagrams; for all distances at which the time-space line passes through a forbidden zone, the vertical profile also passes through a forbidden zone and vice-versa.

To illustrate this, Figure 6 shows an example of coupled forbidden zones. It can be seen that there will be two real conflicts along the observed aircraft's trajectory. Note that the TSD also shows two potential conflicts in the time-space domain, and the VSD shows one potential conflict in the vertical dimension. The figure shows how the along-track distances of forbidden zones due to real conflicts in the TSD and VSD relate. The mutual distances are indicated in red along the distance axis (this is not shown in the interface). Furthermore, the forbidden zones in the VSD provide additional information about the vertical nature of the conflict; the first conflict is caused by a crossing aircraft at the same altitude, and the second conflict is caused by a faster descending aircraft close to the fix. 


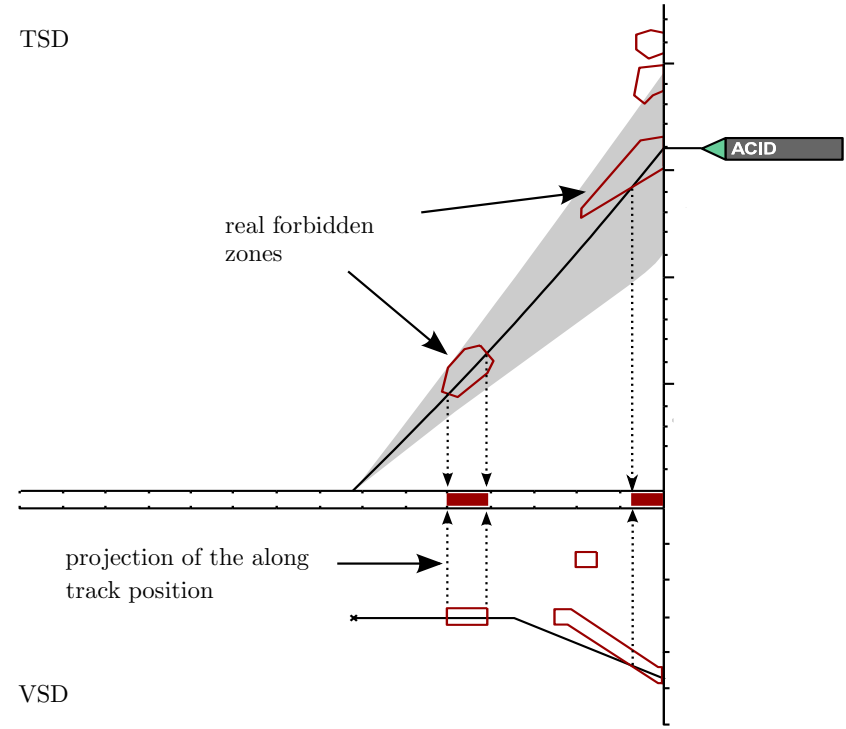

Figure 6: Example of coupled forbidden zones on the TSD and VSD

\section{Idle descent assumption}

In the previous research, for the calculation of the four-dimensional trajectories, a constant descent angle of two degrees towards the IAF was assumed. This ensured that an aircraft could fly along a 4DT with all speeds within its speed envelope. However, with this relatively shallow angle, the descent of the aircraft stretches over a long ground track. When considering the size of an average ACC-sector in Western Europe, in most cases, the descent would already have to be initiated in the adjacent sector. This type of descent leaves little room for manipulating the vertical profile in the own sector. Secondly, when all aircraft descend with the same angle to a single point (in this case, the IAF), the traffic movements are restricted to a (sloped) two-dimensional plane. Resolving conflicts or delaying aircraft by means of path stretching is then more likely to result in a new conflict with other traffic.

To enable the manipulation of the vertical profile, and to let the aircraft move out-of-plane, a new type of descent has been used in the current research. The aircraft are assumed to cruise at their initial flight level until top of descent (TOD) to the IAF. The aircraft will then descend towards the fix with idle thrust, and at a constant Indicated Airspeed (IAS). As a consequence, the aircraft will descend with their maximum rate of descent (ROD) at a given IAS. Note that such descents are frequently performed during current night-time operations in the Amsterdam FIR for reasons of noise abatement.

This assumption however constrains the minimum feasible speed at which the aircraft can fly along the 4DT by the required ground track to perform the descent. In general, most aircraft can descend at a steeper angle when flying at a higher IAS (for all altitudes). Consequently, the required ground track to descend is shorter with a higher speed. Now assume that an aircraft is close to its TOD, and is flying at its maximum IAS. Any speed reduction would cause the aircraft not to meet the altitude constraint placed at the fix (FL70 for the current research). This is implicitly taken into account during the calculation of the feasible time-space lines in the TSD; when an aircraft is close to its top of descent, the controller will have little options to slow down the aircraft.

\section{B. Improved manipulation of the 4DT}

The overall purpose of the interface is to support the ATCo with maintaining a safe and efficient inbound planning. In order to cope with delays, and for reasons of separation, the controller must therefore be able to manipulate the planned trajectory of each individual aircraft. In current ATC operations, an ATCo can issue vector instructions to change an aircrafts present speed, route/heading or altitude. When considering the manipulation of a 4DT however, each renegotiation of a previously agreed trajectory will always result in the generation of a new 4DT; a single change 
will have an effect along the entire trajectory. Because of the complexity of this task, the interface has been designed as a direct manipulation interface (DMI). ${ }^{21}$ By direct graphical manipulation of certain 'goal variables', the complete trajectory will be recalculated and the implications will immediately be visualized to the controller. Consequently, the controller is then able to perceptually determine if their action is useful, and furthers their goal.

The choice for the 'arrival time at the fix' as a goal variable to manipulate (rather than speed directly) was reported to be a pleasant method of working, and therefore will remain unchanged. However, in order to provide the ATCo with more freedom of control, the interface has been enhanced to support direct manipulation of the vertical profile. Secondly, the route control has been expanded, providing the controller with more freedom to plan the horizontal path.

\section{Direct manipulation of the vertical profile}

When a conflict cannot be resolved by a change in speed alone, separating the aircraft vertically is often a more preferred option than re-routing them from their originally planned horizontal path. This not only prevents the spreading of trajectories within the sector, but also has less of an impact on the aircrafts planned arrival time at the fix.

The interface supports this by letting the controller select an intermediate altitude on the VSD, by means of direct manipulation, to which the selected aircraft will descend. Note that only descents are considered in the current research. The trajectory is then immediately recalculated with the following implications:

- The vertical profile is recalculated such that the aircraft descends (with idle thrust) to the intermediate altitude, after which it will continue its cruise until top of descent to the fix;

- The selected time-space line will slant upwards slightly due to the lower ground speed caused by the lower altitude (assuming a constant IAS speed profile);

- Forbidden zones in the TSD can disappear because the path of a certain aircraft will no longer be crossed; and

- New forbidden zones could appear on the TSD because the paths of different aircraft will be crossed.

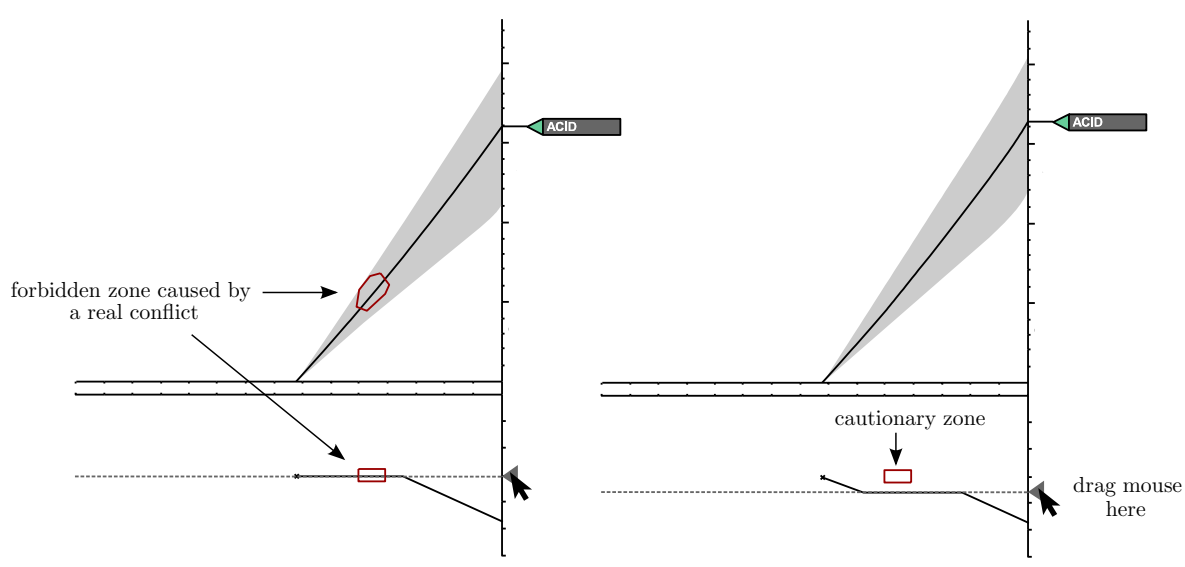

Figure 7: Conflict resolution by separating aircraft in the vertical plane

This principle is visualized in Figure 7. On the left side, the TSD and VSD of the selected aircraft is shown. It can be seen that the time-space line and vertical profile pass through a forbidden zone; a loss of separation will occur at a future time if nothing is done. In this case, a resolution strategy could be to let the selected aircraft descend. This can be done by dragging an indicator triangle, located at the right hand side of the VSD, downwards to a new altitude. The consequences of this action are shown on the figure to the right. As a result, the forbidden zone in the TSD will disappear because the trajectories of both aircraft will no longer truly intersect. The forbidden zone in the VSD then becomes a cautionary zone, indicating that an aircraft will pass overhead, but will not cause an actual loss of separation. 


\section{Improved manipulation of the horizontal route}

In the previous research, the horizontal path of an aircraft could be stretched by either changing the arrival fix, or re-routing it around a single point within the sector. The previous validation experiment showed that in case of considerable delays, some aircraft would have to be significantly re-routed from their original paths. Consequently, the wide spread of trajectories often caused confusion; if a controller would reroute an aircraft to solve one conflict, it was likely that a new conflict would be created.

Therefore, the options to stretch the path of an aircraft have been expanded in the current research. Changing the arrival fix is still supported, but instead of re-routing aircraft through a single point, a set of waypoints can be created (only inside the own sector), fully defining the aircraft's horizontal route to the fix. Waypoints can be added, deleted, and manipulated on the plan view display by means of direct manipulation. When a waypoint is added, it can be dragged and dropped at a new location, after which the trajectory will immediately be re-calculated and visualized to the controller. This provides the ATCo with a method to create organized streams of traffic, which resembles the current method of working, and therefore, could be beneficial for situation awareness.

\section{Consistency and reversibility of control}

The three goal variables which now can be manipulated with the interface are the aircrafts speed profile (a constant IAS profile which is calculated from the selected arrival time at the fix), an intermediate altitude (which is selected on the VSD) and a set of waypoints which define the aircrafts horizontal route. From this input, a trajectory calculator can determine the complete 4DT. For the reason of consistency and clarity, the manipulation of one element should not influence a previous instruction; e.g. a change to the aircrafts horizontal route should not change the selected speed. As the three goal variables are now represented on three different areas on the interface, these are decoupled, and can be manipulated on their own representation; the route on the PVD, the vertical profile on the VSD, and the speed on the TSD (Figure 8). Each manipulation will therefore not have an influence on the other input parameters.

Secondly, in order to let the controller investigate the various solutions which are possible for solving a situation, after each manipulation, a preview of the new trajectory is calculated and visualized on the interface. Note that this preview is only available to the controller, and meanwhile the aircraft will continue along its original path. When the controller is satisfied with the new plan, it can be sent to the aircraft for execution. If the ATCo is not, the plan can be discarded. This type of reversibility of operations for a DMI has shown to be important to reduce the controllers anxiety of working with the interface. ${ }^{21}$
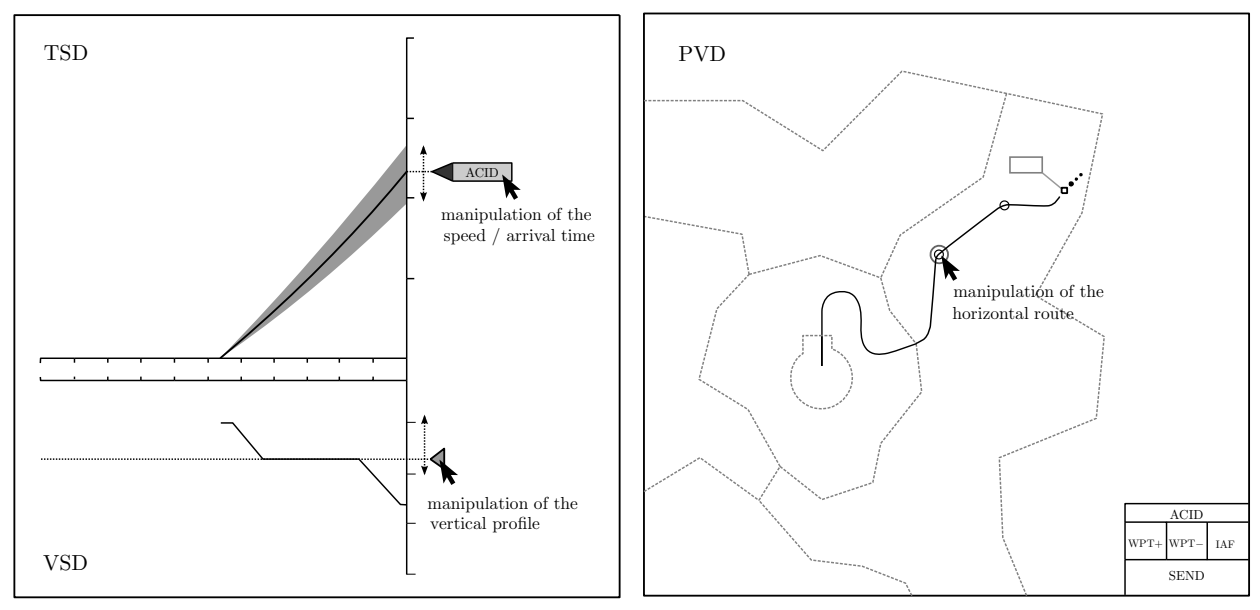

Figure 8: Direct manipulation of the route (PVD), speed (TSD) and vertical profile (VSD) for a selected aircraft

\section{Integration of information across screens}

In the previous research, mapping information between the planning interface and the spatial situation on the PVD was reported to be difficult. As a consequence, the fact that the controllers often used the TSD as the primary display 
to manage the traffic frequently resulted in a poor level of situation awareness. With the addition of the VSD, more information is provided with respect to an aircrafts planned vertical profile. However, the information shown on the TSD and VSD still does not have a clear relationship with the spatial traffic situation.

The importance of integrating data across successive displays has been underlined by the concept of visual momentum. ${ }^{22}$ Visual momentum is best described as the user's ability to extract and integrate information across displays to create a stable percept of the environment. A good level of continuity and consistency between displays is necessary to support the rapid comprehension of the presented data, and could reduce the controllers' mental effort.

Following the framework offered by this concept, two new tools have been added to the interface, purposely designed for integrating related across-screen information. These are:

- Conflict visualization on the PVD, which provides information about the spatial location of conflicts, and

- The 'ghost view' tool, with which the planned future positions of the aircraft can be projected onto the PVD.

\section{Conflict visualization on the PVD}

The forbidden zones on the TSD and VSD provide information about the nature of conflicts in the time-space and vertical dimension. Although the along-track distance of these zones can be read from both diagrams, the information is difficult to relate to a spatial location on the PVD. This was confirmed in the previous validation experiment by the fact that almost all subjects reported that it was difficult to interpret the forbidden zones.

The interface supports this across-screen translation by graphically indicating the section of a selected aircrafts trajectory which will be in conflict with other traffic on the PVD. Note that this section relates to the along-track distances at which the time-space line and vertical profile pass through a forbidden zone in the TSD and VSD respectively. When the trajectory of the conflicting aircraft is also visualized, this can provide a cue to how the conflict could be resolved by a reroute command.

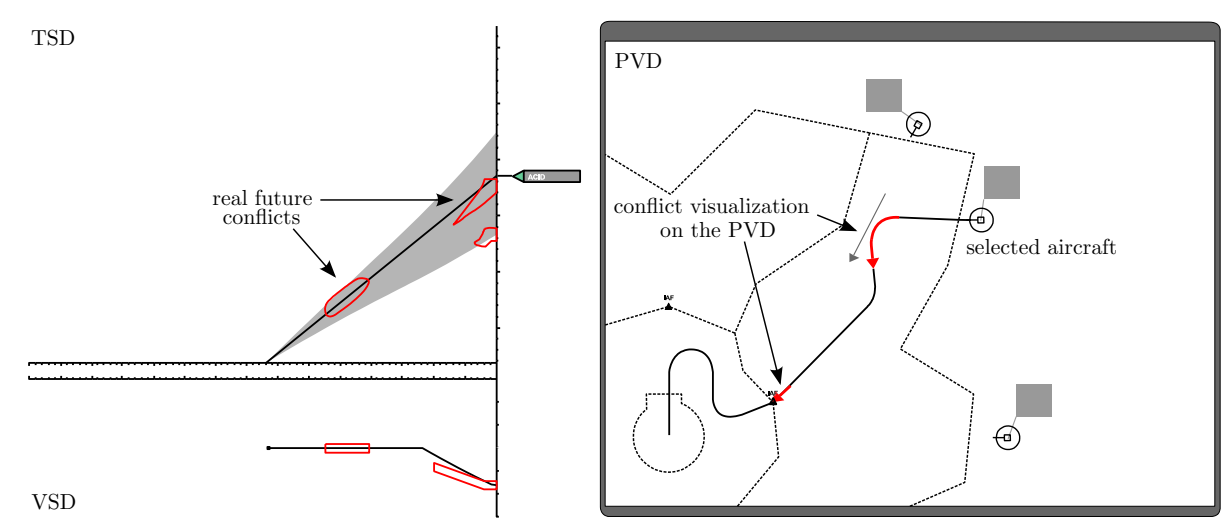

Figure 9: Translation of forbidden zones to a spatial location on the plan view display

Figure 9 shows how this principle works. The TSD and VSD show that there will be two conflicts along the trajectory of the selected aircraft. The first conflict occurs during level flight, and the second occurs close to the fix (whilst descending). The PVD is shown on the right side of the figure with the section of conflicting trajectory highlighted. The red line relates to the occupied section of the trajectory of the selected aircraft. The second (light gray) line indicates the trajectory of the conflicting aircraft. Arrow heads are added to indicate the direction of flight. Consequently, this information can be used as a basis for a resolution strategy. For the situation in the figure: the controller could choose to let the selected aircraft fly directly towards the fix after entering the sector, resolving the first conflict. Most probably, a following change in speed could then resolve the second. The conflict visualization is updated after each recalculation and manipulation of the trajectory. As a result, it can immediately be seen if a new trajectory will result in new conflicts without having to consult the TSD or PVD. 


\section{Ghost view tool}

With the previously developed interface there was no possibility to observe how the traffic situation would evolve in time, otherwise than monitoring and making a mental projection. The 4DT of a single aircraft could be visualized on the TSD and PVD (when selected), but no information was available about the future spatial interaction with other traffic. The previous validation experiment showed that when the amount of traffic was high and many aircraft had to be re-routed, it became a difficult task to fully comprehend how the traffic picture would evolve.

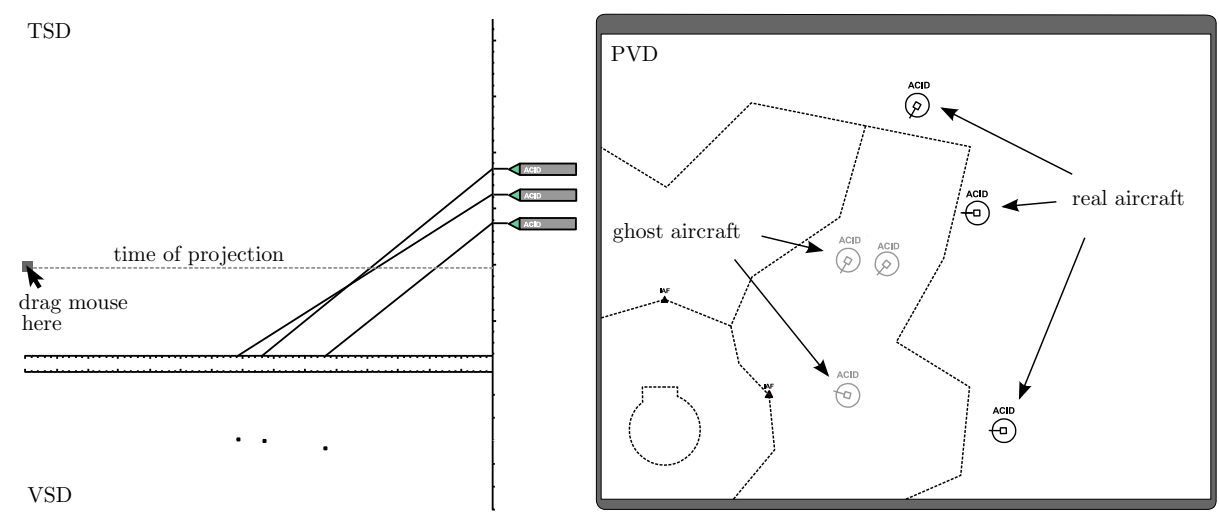

Figure 10: Principle workings of the ghost view tool

To support the ATCo with creating a mental projection, a so called ghost view tool has been added to the interface. By dragging a slider, located at the left hand side of the TSD, up and down along the time-axis, a dynamical projection of the future traffic situation is made on the PVD; a duplicate ghost image of each aircraft is then visualized on the PVD for the selected time (Figure 10). Each ghost image is labeled with the ACID for identification, and the protected zone is also shown. The tool can support the ATCo with making a global plan for the inbound traffic. For instance, by viewing the planned movements of the traffic, a logical inbound sequence can be determined, or areas in the sector which are safe to re-route aircraft through can be found. Secondly, the tool can also support the controller with the manipulation of individual trajectories; after each manipulation to speed, route or vertical profile, the ghost position of the selected aircraft is updated. The controller can then immediately see how the new trajectory interacts with the other traffic.

Now that the enhancements to the three main areas of focus have been addressed, the actual implementation of interface in a PC-based simulation program will be discussed.

\section{PC-based implementation}

This section describes the PC-based implementation of the interface which has been built for the validation experiment. From now on the re-designed interface will be referred to as the Time-Space and Vertical Display (TSVD).

Figure 11 shows a detailed sketch of the TSVD and the PVD when an aircraft is selected. On the PVD, a topdown visualization of the controlled airspace, indicating sector borders, IAFs and waypoints is provided. Note that the controller can zoom in and out, or move the center of view to a new location by using the mouse or the buttons in the bottom left corner. Furthermore, the current aircraft positions are shown on the PVD and updated every four seconds. Each aircraft has a (draggable) label attached, showing ACID, current flight level, aircraft type, indicated airspeed, ground speed and the active IAF through which they will enter the TMA. In addition, the visualization of history dots, a speed vector and the protected zone can be turned on or off, depending on the controllers preference. The selected aircraft is highlighted in white, and its planned horizontal route is shown by a white dotted line. The PVD also shows the active conflicts along the trajectory of the selected aircraft. Next to the airspace and aircraft elements, a clock showing the current (simulation) time is visualized in the top left corner the PVD. Notifications and warnings are shown in the top right corner to notify the controller of certain events. For instance, a message is shown when an aircraft enters or leaves the active sector, or confirms having received its new flight plan. 
The TSVD is shown behind the PVD in the figure. However, during the actual experiment the TSVD and PVD were displayed on separate screens. The time axis of the TSD represents the time from 'now' up to thirty minutes in the future and the current hour is displayed beneath. The 'distance to the IAF' axis shows distances up to $150 \mathrm{NM}$ from the fix and the altitude axis of the VSD indicates flight levels up to FL300. Furthermore, a grid can be visualized along all axis of the TSVD, and a second clock is shown in the left top corner. The labels of all aircraft inside the controlled sector, or within 18 minutes from the fix are shown at their respective arrival time (along the time axis). The reason for the 18 minutes criterion is that for an ACC-ATCo in the Amsterdam FIR, inbound traffic usually first appears on the radar screen approximately 18 minutes before being handed-off to the APP controller (which is responsible for controlling the airspace inside TMA). The label contains the aircraft ID and the delay time in minutes with respect to the initial arrival time at the fix. In addition, the labels are placed in the column beneath the approach fix over which they are planned to enter the TMA. The label, time-space line and vertical profile of the selected aircraft are highlighted in white. The speed constraints and margin of arrival times are visualized by the light gray triangular area on the TSD. As will be explained in the following section, during the experimental validation the controller was either allowed, or not allowed to manipulate an aircraft in the adjacent sector. Therefore, only one triangle is shown. The forbidden zones for the selected aircraft are visualized as red circumvented areas. When a zone is clicked on, it will be highlighted and the ACID of the conflicting aircraft will appear next to it. The 'blue bars' which indicate the wake-vortex separation at the runway are also shown. In the TSVD, the blue bars do not shift to right when they overlap as in the interface designed in the previous research. Instead, the overlapping area changes color to a lighter shade of blue (this is not shown in the figure).

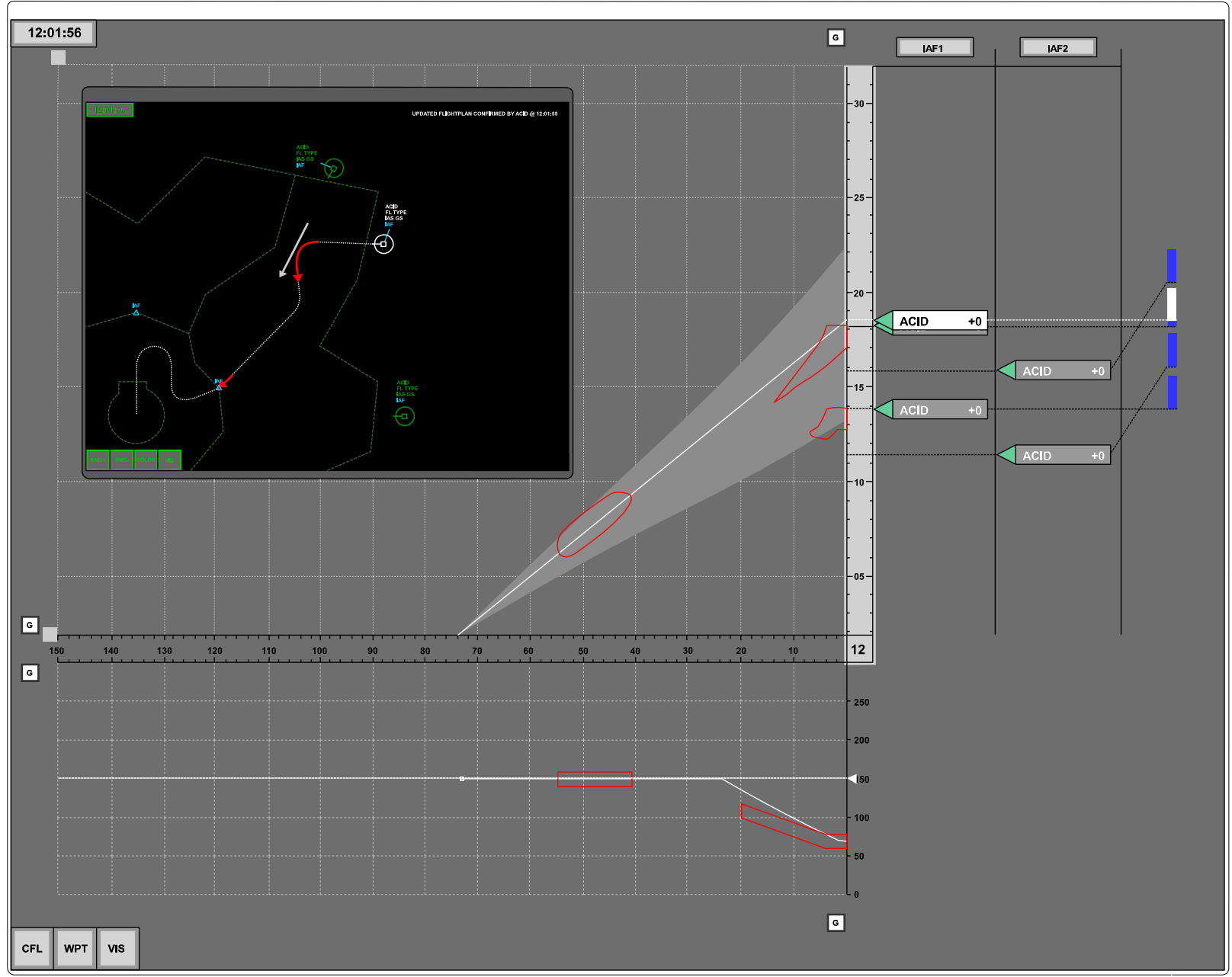

Figure 11: Detailed sketch of the TSVD when an aircraft is selected

By clicking on the label of the selected aircraft a second time (either on the TSVD or PVD), the interface will enter the 'manipulation' mode. The white color of the selected aircraft will then change to yellow as a visual indication. In 
this mode, the controller can manipulate the 4DT of the aircraft. The route control buttons and the button with which the ATCo can send the manipulated trajectory to the aircraft will then appear in the right bottom corner of the PVD. This was purposely done such that the controller has to focus their attention on the spatial situation on the PVD before sending the trajectory.
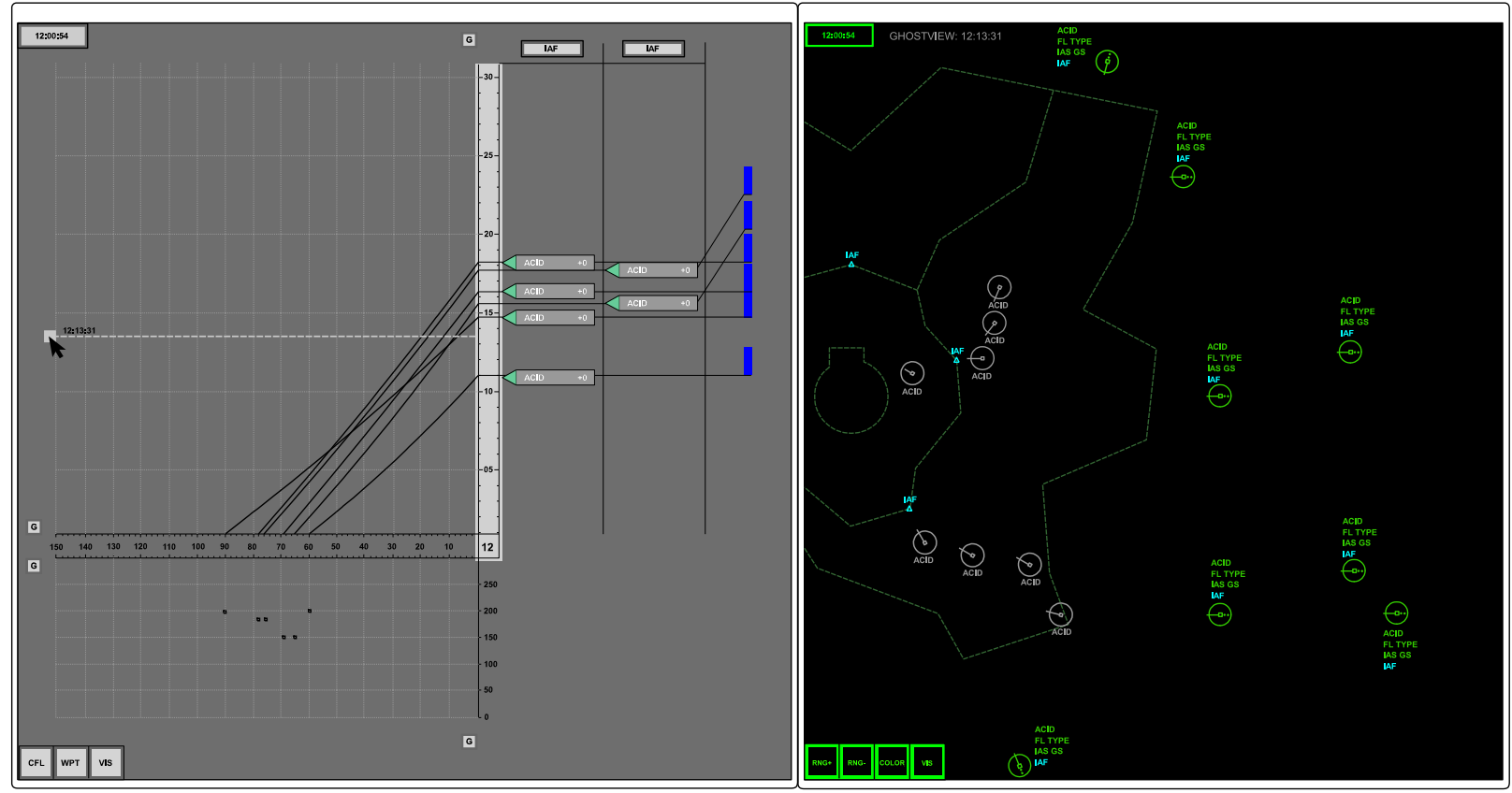

Figure 12: Detailed sketch of the TSVD and PVD when no aircraft is selected, with the ghost view tool active

An aircraft can either be deselected by pressing the label again, or by pressing the a dedicated key on the keyboard. Figure 12 shows a second detailed sketch of the TSVD when no aircraft is selected. The time-space lines of all aircraft are shown in the TSD. The current flight level of each aircraft is indicated on the VSD by a square located beneath the current position. In this figure, the blue bars are neatly stacked, indicating a favorable situation inside the TMA. Furthermore, the ghost view tool is active, and the slider is dragged to a time approximately twelve minutes in the future. The exact time of projection is shown above the ghost slider indicator line (on the TSD), and again in the top left corner of the PVD. The projected ghost images of the aircraft are visualized on the PVD.

\section{Experiment}

With the PC-based implementation of the TSVD, an experimental validation has been performed. Twelve test subjects participated in this experiment to answer the following questions:

- Does the addition of the new tools (conflict visualization on the PVD and the ghost view tool) improve situation awareness and reduce controller workload?

- What is the effect of the possibility to issue speed requests to aircraft before they physically enter the controlled sector to the controller situation awareness, workload and the efficiency of the overall traffic flow?

- What is the overall appreciation of the TSVD, and what should be focused on during a further development process?

\section{A. Method}

The experiment has been performed with a dedicated ATC-simulation program, running on a single laptop computer. The subjects were given four different scenarios to solve in the same sector, in four different conditions. During each run, the overall goal was to plan and manage the 4DTs of the inbound traffic safely (without loss of separation) through the controlled ACC-sector to the entry points of the TMA. Secondly, the arrival times of the aircraft at the IAF had to 
be planned such that no loss of separation would occur inside the TMA. After initialization, the subjects were free to solve the scenarios by issuing changes to the speed, route and vertical profile of the 4DTs of the aircraft. Because the trajectories were generated and executed automatically, no additional input was required from the experimenter.

\section{Apparatus}

The PVD was presented on a 17-in. wide-screen laptop monitor (60Hz LCD, 1900x1200 resolution). The TSVD was presented on a separate 17-in. screen (75Hz TFT, 1280x1024 resolution), which was placed on the left hand side of the PVD. The controller input was given by a standard mouse, which could traverse between both screens. An overview of the experiment set-up is given in Figure 13.

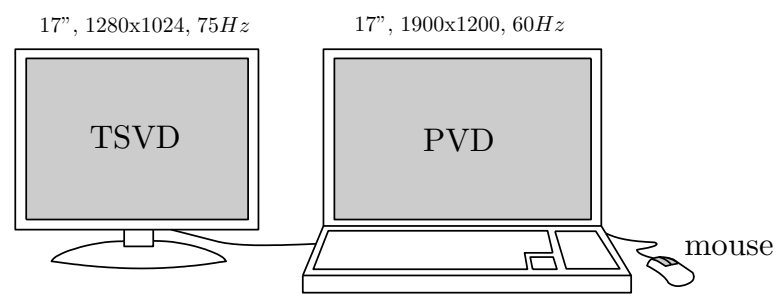

Figure 13: The experiment set-up

\section{Subjects}

The experiments were performed by a total of twelve subjects, divided into three groups. The first group consists of four certified ACC-ATCos, who currently execute radar ATC in the CTA sectors in the Amsterdam FIR. The second group consists of four 'retired' ATCos, two of them former military (DutchMil), and the other two civil. The last group consists of four people who have never actively controlled air traffic, but do perform work in the ATC-domain. Several participants have also participated in the previous validation experiment. Table 1 shows the details.

Table 1: Details of the test subjects who participated in the experiment

\begin{tabular}{llll}
\hline & function & experience & age \\
\hline ATCos & & & \\
\hline 1 & ACC-ATCo & 7 years & 30 \\
$2^{*}$ & ACC-ATCo & $4 \frac{1}{2}$ years & 28 \\
$3^{*}$ & ACC-ATCo & 21 years & 44 \\
$4^{*}$ & ACC-ATCo & 16 years & 35 \\
\hline retired ATCos & & & 42 \\
\hline $5 *$ & former military ATCo & 6 years & 56 \\
6 & former civil ATCo & 30 years & 59 \\
7 & former civil ATCo & 33 years & 44 \\
$8^{*}$ & former military ATCo & 5 years & 29 \\
\hline non ATCos & & & 47 \\
9 & employee LVNL & 5-day ATC course \\
10 & employee DUT & 5-day ATC course & 42 \\
11 & employee DUT & 5-day ATC course & 25 \\
12 & MSc. graduate DUT & previous graduation work & 25 \\
\hline \hline
\end{tabular}

* also a participant of the initial validation experiment

\section{Scenarios}

The subjects were asked to manage the traffic in a hypothetical sector, in four different traffic scenarios. Each scenario presented a total of 17 inbound aircraft entering the sector from the north, east and south. In total each participant had 
to control $(17 \times 4=) 68$ aircraft. Outbound and transit aircraft were not considered during the experiment. Furthermore, all scenarios were approximately of an equal level of difficulty.

Three types of aircraft were simulated in clean configuration, each with different performance characteristics which influence the feasible range of 4DTs: The Airbus A319-114, the Airbus A330-301 and the Airbus A340-313. The Eurocontrol GAME ${ }^{23,24}$ aircraft performance model has been used to calculate the relevant elements of aircraft performance such as minimum/maximum speed and idle rate of descent.

The aircraft entered the controlled sector between FL120 and FL230, with a IAS varying between 220kts and 280kts, and were given an initial 4DT leading to one of two IAFs. For reasons of simplification, a fixed turn radius of 5NM has been assumed, and the fixed minimum landing interval between two aircraft (length of the 'blue bars') has been set to 1.7 minutes for all aircraft. The initial conditions were set such that at a certain point the TMA would become congested and the subject would have to issue changes to the 4DTs to maintain a safe and efficient sequence of aircraft.

\section{Independent variables}

For each test subject, the sequence of the scenarios was always the same, whilst the sequence of conditions varied. Two variables defined the four conditions in which the traffic had to be controlled. These were:

- TOOLS: The possibility to use the ghost view tool and the conflict visualization on the PVD, and;

- SECTOR: The possibility to issue speed requests to aircraft in the adjacent sector.

The TOOLS could either be enabled or disabled. When enabled, the controller could use the ghost view tool, and conflict visualization on the PVD was enabled. These features would not be available when the TOOLS were disabled.

The SECTOR option is defined by the ability to control the speed of an aircraft in the adjacent sector. When enabled, the 'manipulation' mode of the TSVD could be entered for all inbound aircraft. Secondly, all aircraft which were closer than 18 minutes from the fix would be displayed on the interface. When disabled, only the aircraft which were physically located inside the active sector could be controlled, and only these aircraft were displayed on the TSVD. Note that all aircraft were always visible on the PVD.

An overview of the four conditions is given in Table 2. Table 3 shows the order in which the conditions were presented to each participant within a separate group. In total, This scheme was repeated three times (once for each group).

Table 2: Definition of the four conditions

\begin{tabular}{lll}
\hline \hline condition & TOOLS & SECTOR \\
\hline $\mathbf{T}_{\mathbf{E}} \mathbf{S}_{\mathbf{E}}$ & enabled & enabled \\
$\mathbf{T}_{\mathbf{E}} \mathbf{S}_{\mathbf{D}}$ & enabled & disabled \\
$\mathbf{T}_{\mathbf{D}} \mathbf{S}_{\mathbf{E}}$ & disabled & enabled \\
$\mathbf{T}_{\mathbf{D}} \mathbf{S}_{\mathbf{D}}$ & disabled & disabled \\
\hline \hline
\end{tabular}

Table 3: Order of the conditions for the within group participants

\begin{tabular}{lllll}
\hline \hline within group participant & \multicolumn{4}{l}{ order of conditions } \\
\hline 1 & $\mathbf{T}_{\mathbf{E}} \mathbf{S}_{\mathbf{E}}$ & $\mathbf{T}_{\mathbf{E}} \mathbf{S}_{\mathbf{D}}$ & $\mathbf{T}_{\mathbf{D}} \mathbf{S}_{\mathbf{E}}$ & $\mathbf{T}_{\mathbf{D}} \mathbf{S}_{\mathbf{D}}$ \\
2 & $\mathbf{T}_{\mathbf{D}} \mathbf{S}_{\mathbf{D}}$ & $\mathbf{T}_{\mathbf{E}} \mathbf{S}_{\mathbf{E}}$ & $\mathbf{T}_{\mathbf{E}} \mathbf{S}_{\mathbf{D}}$ & $\mathbf{T}_{\mathbf{D}} \mathbf{S}_{\mathbf{E}}$ \\
3 & $\mathbf{T}_{\mathbf{D}} \mathbf{S}_{\mathbf{E}}$ & $\mathbf{T}_{\mathbf{D}} \mathbf{S}_{\mathbf{D}}$ & $\mathbf{T}_{\mathbf{E}} \mathbf{S}_{\mathbf{E}}$ & $\mathbf{T}_{\mathbf{E}} \mathbf{S}_{\mathbf{D}}$ \\
4 & $\mathbf{T}_{\mathbf{E}} \mathbf{S}_{\mathbf{D}}$ & $\mathbf{T}_{\mathbf{D}} \mathbf{S}_{\mathbf{E}}$ & $\mathbf{T}_{\mathbf{D}} \mathbf{S}_{\mathbf{D}}$ & $\mathbf{T}_{\mathbf{E}} \mathbf{S}_{\mathbf{E}}$ \\
\hline \hline
\end{tabular}




\section{Dependent measures}

The dependent measures which have been used to analyze and compare the four conditions were:

- Loss of separation. Loss of separation was measured by logging the number of separation violations between aircraft. For the experiment, the minimum separation criteria were set to 5NM horizontally and 1,000ft vertically.

- Workload. The controller workload was measured by using a digital version of the NASA-Task Load Index $\left(\right.$ NASA-TLX ${ }^{25}$ ) subjective mental workload questionnaire. The TLX-questionnaire returns a score ranging between 0 and 100. Here, a higher score indicates a higher workload.

- Situation Awareness. The controller situation awareness was measured using the Eurocontrol SASHA ${ }^{26}$ questionnaire, which provides an overall subjective score for SA. The scores range between 0 and 6 . A higher score indicates a higher (better) level of SA.

- Logged data. During each run an extensive log was generated of the individual aircraft movements and the human interaction with the interface.

- Condition questionnaire. The participants were asked to self-grade their performance and their use of the interface by means of six multiple choice questions. Answers were given on a six level Likert scale

\section{Final questionnaire}

A final questionnaire regarding the overall use and appreciation of the TSVD was presented to the subjects after the completion of all runs. The participants were asked to grade certain functionalities of the interface, and were given the possibility to add additional comments for each question.

\section{B. Procedure}

The experiment started with a 45-minute briefing in which the basic working principles of the interface were discussed and demonstrated. This included the use of the TSD, VSD, PVD and TMA-separation bars (blue bars) to control the 4DTs of the traffic safely and efficiently. Secondly, the interpretation of the forbidden zones on the TSD and VSD, conflict zones on the PVD, and the use of the ghost view tool were explained in more detail. For this purpose, four training scenarios were available, in which the TOOLS and SECTOR options were always enabled. The level of difficulty progressed as the briefing continued (higher aircraft count and bunching), ending up with a scenario similar to the ones presented during the actual experiment. The briefing ended when the subjects indicated that they had a good understanding of the interface.

After the briefing, the subjects were told that the use of the TOOLS and SECTOR options would vary, depending on the condition. The actual initialized condition was mentioned before each run. After the initial conditions were set, the subjects were left free to control the traffic and use the interface as they wanted. During the experiment, the subjects were also allowed to fast-forward the simulation (speed $\times 10)$ when they had the feeling that all aircraft were safely controlled, or they had to wait for a next aircraft to enter the sector. Depending on the condition, the duration of the scenarios ranged between 10 and 30 minutes. In total, the duration of an experiment, including briefing and final questionnaire, ranged between two and two and a half hours.

\section{Hypotheses}

Next to the overall validation of the TSVD, the experiment was performed to investigate the validity of the following hypotheses regarding workload and situation awareness.

\section{Workload}

The ghost view tool and the conflict visualization on the PVD are purposely designed to reduce the controllers' mental effort to interpret and translate information visualized on the TSVD to spatial locations on the PVD. Therefore, workload is hypothesized to reduce when the TOOLS option is enabled. When the SECTOR option is enabled, the controller will be able to separate aircraft and plan the sequence of traffic further ahead in time. As a result, especially the temporal demand of ATC task is expected to be reduced. 


\section{Situation awareness}

The ghost view tool has been designed with the purpose to directly visualize the (planned) time-evolution of the traffic flow to the controller. As a result, situation awareness is hypothesized to increase because interpretation and anticipation are performed for the controller. Secondly, conflict visualization on the PVD provides the controller with a spatial interpretation of the forbidden zones shown on the TSD and VSD. As the visualization of conflicts is more complete, an increase of situation awareness is expected. Therefore, an overall increase of situation awareness is hypothesized with the TOOLS enabled. When the SECTOR option is enabled, the horizontal trajectories of aircraft are expected to become more predictable; path stretching in the own sector could largely be avoided by speed changes to aircraft in the adjacent sector. Creating a mental projection is expected to require less cognition, and therefore situation awareness is hypothesized to increase.

\section{Feedback and Results}

After the experiment, a detailed analysis of the experimental feedback and results has been conducted. The most important feedback and results will be discussed in this section.

\section{A. Loss of separation}

A detailed analysis of the logged trajectories of all $(12 \times 4 \times 17=) 816$ controlled aircraft showed that no actual loss of separation occurred inside the controlled sector at any point during the experimental runs.

\section{B. Workload}

Figure 14(a) shows a boxplot of the measured TLX scores per condition, and per subject group. The figure shows that the lowest workload scores attribute to the ATCo group, and the highest to the non ATCo group. Firstly, by using a Kolmogorov-Smirnov test, the TLX data were found to be normally distributed $(D(48)=0.120, p>.05)$. A repeatedmeasures Analysis of Variance (ANOVA) of the TLX scores showed that the between-group effect was not significant $(F(2,9)=0.627, p=0.56)$. Therefore, the within-subject effects due to the TOOLS and SECTOR option have been tested for all participants combined.

Figure 14(a) shows that the mean workload scores for the conditions with TOOLS enabled are lower than for the conditions with the TOOLS disabled for all groups (by comparing conditions $\mathbf{T}_{\mathbf{E}} \mathbf{S}_{\mathbf{E}}$ and $\mathbf{T}_{\mathbf{D}} \mathbf{S}_{\mathbf{E}}$, and conditions $\mathbf{T}_{\mathbf{E}} \mathbf{S}_{\mathbf{D}}$ and $\left.\mathbf{T}_{\mathbf{D}} \mathbf{S}_{\mathbf{D}}\right)$. However, the within-subject effects showed no significant decrease in workload $(F(1,9)=1.760$, $p=0.17)$. The results did show a significantly lower workload for the conditions with the SECTOR option enabled, compared to the conditions with the SECTOR option disabled $(F(1,9)=11.087, p<0.01)$.

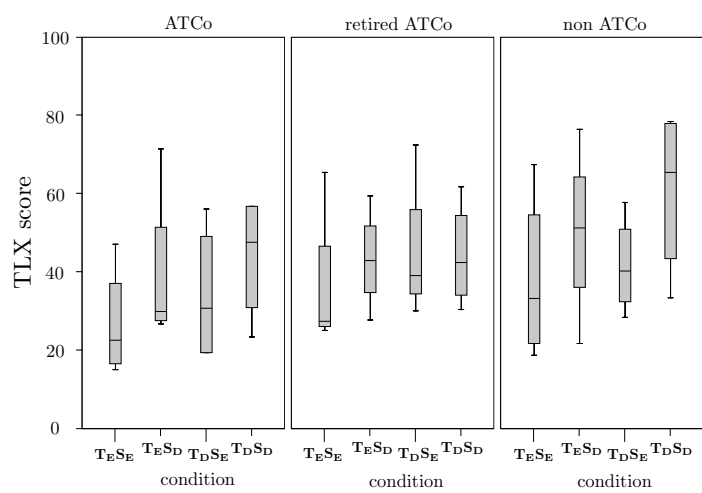

(a) Boxplot of the measured TLX scores

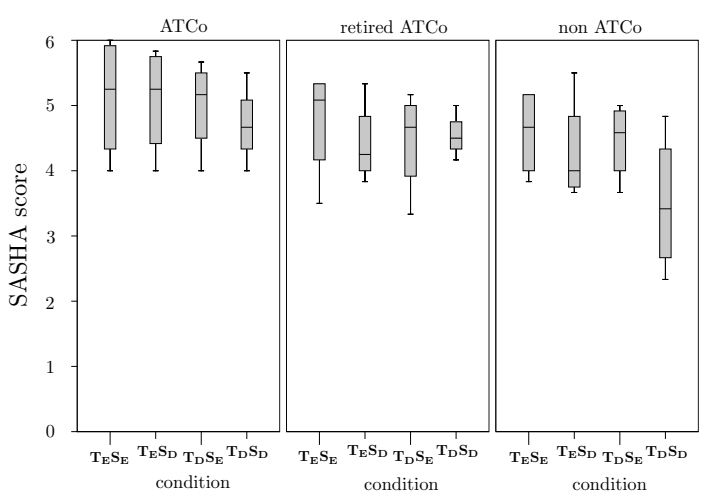

(b) Boxplot of the measured SASHA scores

Figure 14: Boxplots of the measured NASA-TLX and SASHA scores per condition, clustered per subject group 


\section{Situation awareness}

Figure 14(b) shows a boxplot of the measured overall SASHA scores. The figure shows that the ATCo group rate their situation awareness the highest (closest to the maximum score of six), and the non ATCo group the lowest. Furthermore, the figure shows that most situation awareness scores are located in the higher range of the scale. A Kolmogorov-Smirnov test was performed, and showed that the measured SASHA data were normally distributed $(D(48)=0.085, p>.05)$. Again, no significant between-group effect was found by using a repeated-measures ANOVA of the SASHA scores $(F(2,9)=1.439, p=0.29)$. By evaluating the within-subject effects of all participants combined, no significant increase of situation awareness was found for the conditions with the TOOLS enabled compared to the conditions with the TOOLS disabled $(F(1,9)=3.416, p=0.098)$. However, situation awareness was significantly higher for the conditions with the SECTOR option enabled, compared to the conditions with the SECTOR option disabled $(F(1,9)=6.153, p<0.05)$.

\section{Correlation analysis}

A bivariate Pearson correlation test has been performed to investigate whether the workload and situation awareness scores are correlated. The results show that situation awareness has a significant negative correlation with workload $(r(46)=-0.859, p<0.001)$. Figure 15 shows a scatterplot of the SASHA scores versus the TLX scores for the four conditions. The figure shows that a lower level of situation awareness correlates with a higher workload. Furthermore, the highest level of SA and lowest workload attribute to condition $\mathbf{T}_{\mathbf{E}} \mathbf{S}_{\mathbf{E}}$, and the opposite to condition $\mathbf{T}_{\mathbf{D}} \mathbf{S}_{\mathbf{D}}$.

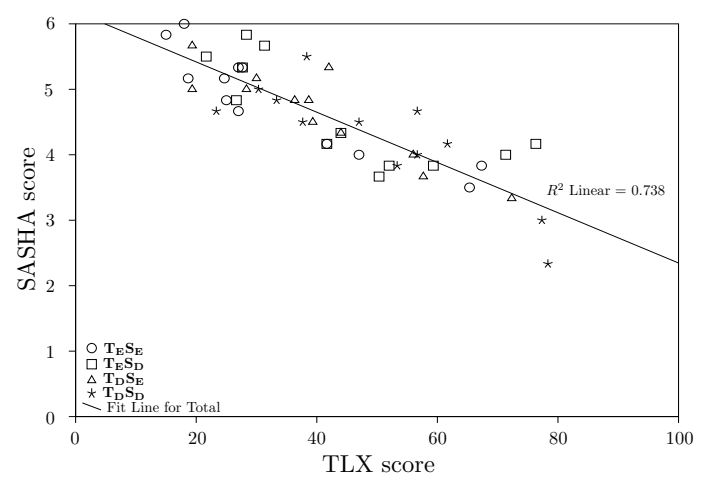

Figure 15: Scatter plot of the TLX versus the SASHA scores

\section{E. Logged data}

The flown trajectory, delay time and number and type of manipulations to the 4DT of each individual aircraft has been logged. Furthermore, data has been collected of the amount of mouse clicks per screen (PVD and TSVD), and of the detailed use of the ghost view tool. The most important findings are reported below.

\section{Aircraft data}

By visually inspecting all logged aircraft trajectories, a clear pattern could be seen between the experimental runs with the SECTOR option enabled and disabled. To illustrate this, Figure 16 shows the logged trajectories of scenario 1 (identical initial traffic conditions) for the ATCo group, controlled under condition $\mathbf{T}_{\mathbf{E}} \mathbf{S}_{\mathbf{E}}$ and condition $\mathbf{T}_{\mathbf{D}} \mathbf{S}_{\mathbf{D}}$. Figure 16(a) shows that almost all aircraft fly directly from the sector border to the fix, indicating that the sequencing of aircraft, and most conflicts, could be resolved by issuing speed requests to the aircraft in the adjacent sector. Figure 16(b) shows that when the aircraft could only be controlled inside the active sector, the paths of more aircraft had to be stretched. By a repeated-measures ANOVA of the number of waypoint manipulations, no significant between subject group effect was found $(F(2,9)=0.934, p=0.428)$. A non-parametric related-samples ranked Friedman test showed a highly significant effect of the number of waypoint manipulations between the four conditions $\left(\chi^{2}(3)=29.161, p\right.$ $<.001)$. This finding was followed up by a Wilcoxon signed-rank test to make a pair wise comparison between the conditions. Table 4 shows the details. The results show that there is no significant effect of the TOOLS to the number of waypoint manipulations (by comparing condition $\mathbf{T}_{\mathbf{E}} \mathbf{S}_{\mathbf{E}}$ and $\mathbf{T}_{\mathbf{D}} \mathbf{S}_{\mathbf{E}}$ and condition $\mathbf{T}_{\mathbf{E}} \mathbf{S}_{\mathbf{D}}$ and $\mathbf{T}_{\mathbf{D}} \mathbf{S}_{\mathbf{D}}$ ). However, 
the number of waypoint manipulations is significantly lower for all conditions where the SECTOR option is enabled compared to conditions where the SECTOR option is disabled. A further analysis showed that in total, the vertical profile of only nine out of the 816 aircraft had been manipulated.

Table 4: Pair wise comparison of the number of waypoint manipulations per condition

\begin{tabular}{|c|c|c|}
\hline conditions & $z$ & asymp. sig. (2-tailed) \\
\hline $\mathbf{T}_{\mathbf{E}} \mathbf{S}_{\mathbf{D}}>\mathbf{T}_{\mathbf{E}} \mathbf{S}_{\mathbf{E}}$ & -2.934 & $p<0.01^{*}$ \\
\hline $\mathbf{T}_{\mathbf{D}} \mathbf{S}_{\mathbf{E}}>\mathbf{T}_{\mathbf{E}} \mathbf{S}_{\mathbf{E}}$ & -1.067 & $p>0.05$ \\
\hline $\mathbf{T}_{\mathbf{D}} \mathbf{S}_{\mathbf{D}}>\mathbf{T}_{\mathbf{E}} \mathbf{S}_{\mathbf{E}}$ & -3.064 & $p<0.01^{*}$ \\
\hline $\mathbf{T}_{\mathbf{D}} \mathbf{S}_{\mathbf{E}}<\mathbf{T}_{\mathbf{E}} \mathbf{S}_{\mathbf{D}}$ & -3.059 & $p<0.01^{*}$ \\
\hline $\mathbf{T}_{\mathbf{D}} \mathbf{S}_{\mathbf{D}}>\mathbf{T}_{\mathbf{E}} \mathbf{S}_{\mathbf{D}}$ & -1.609 & $p>0.05$ \\
\hline $\mathbf{T}_{\mathbf{D}} \mathbf{S}_{\mathbf{D}}>\mathbf{T}_{\mathbf{D}} \mathbf{S}_{\mathbf{E}}$ & -3.061 & $p<0.01 *$ \\
\hline
\end{tabular}

* significant $(p<0.01)$

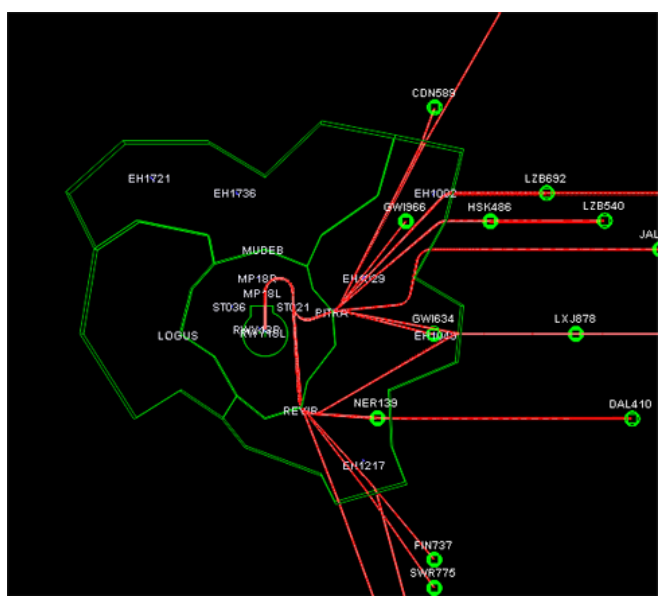

(a) ATCo participant 1, condition $\mathbf{T}_{\mathbf{E}} \mathbf{S}_{\mathbf{E}}$

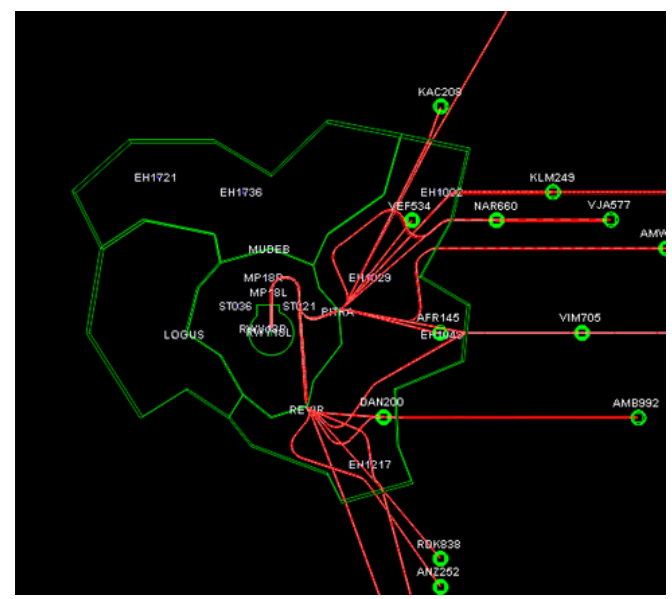

(b) ATCo participant 2, condition $\mathbf{T}_{\mathbf{D}} \mathbf{S}_{\mathbf{D}}$

Figure 16: Logged trajectories of scenario 1, under different conditions

Furthermore, the aircraft delay times with respect to their initial 4DT have been logged. The delay times have been calculated by subtracting the planned arrival time at the runway of the initial 4DT from the planned arrival time of the 4DT at the end of the run. The zero delay cases indicate the aircraft of which the 4DT has not been manipulated. Figure 17(a) shows the cumulative frequency of the delay times per condition, for all controlled aircraft. The figure shows that the aircraft have been delayed the least with both the TOOLS and SECTOR options enabled (condition $\mathbf{T}_{\mathbf{E}} \mathbf{S}_{\mathbf{E}}$ ), and the most with both TOOLS and SECTOR options disabled $\left(\mathbf{T}_{\mathbf{D}} \mathbf{S}_{\mathbf{D}}\right)$. The cumulative distributions of delay for conditions $\mathbf{T}_{\mathbf{E}} \mathbf{S}_{\mathbf{D}}$ and $\mathbf{T}_{\mathbf{D}} \mathbf{S}_{\mathbf{E}}$ lie close together, although, it can be seen that more aircraft could be advanced to arrive earlier than initially planned for condition $\mathbf{T}_{\mathbf{D}} \mathbf{S}_{\mathbf{E}}$.

Figure 17(b) shows a boxplot of all recorded aircraft delay times per condition, and per group. The figure shows that there is a relatively large standard deviation and that there are multiple outliers, indicating aircraft of which the planned arrival time has significantly been advanced or delayed. The average delay time per condition has been calculated for each participant. By means of a Wilcoxon test, a pair wise comparison of the delay per condition showed that only the average delay of condition $\mathbf{T}_{\mathbf{E}} \mathbf{S}_{\mathbf{E}}$ is significantly less compared to condition $\mathbf{T}_{\mathbf{D}} \mathbf{S}_{\mathbf{D}}(z=-2.118, p<0.05)$.

\section{Mouse clicks}

Figure 18(a) shows a boxplot of the mouse click percentage on the TSVD for the four conditions, and per subject group. This percentage is given by the number of clicks on the TSVD with respect to the total amount of clicks on the TSVD and PVD combined. This data has been used as a rough indicator to find out on which screen the attention of the participants was mainly focused. The figure shows that for all subject groups, the mean click percentage is higher for the conditions with the SECTOR options enabled $\left(\mathbf{T}_{\mathbf{E}} \mathbf{S}_{\mathbf{E}}\right.$ and $\left.\mathbf{T}_{\mathbf{D}} \mathbf{S}_{\mathbf{E}}\right)$, compared to the conditions with the 


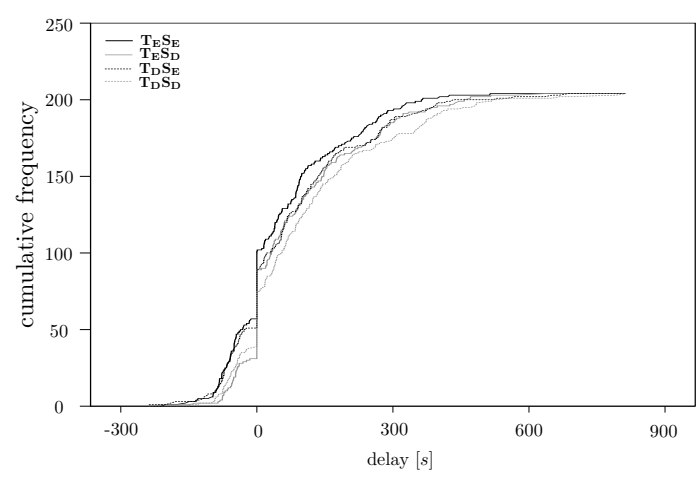

(a) Cumulative frequency of aircraft delay times

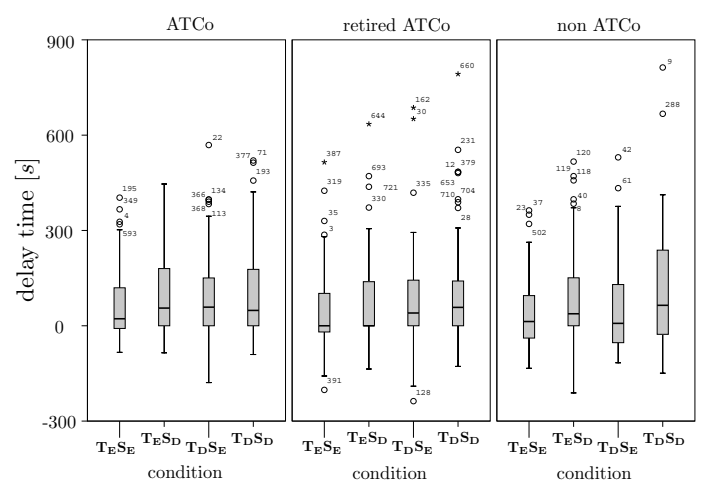

(b) Aircraft delay time boxplot

Figure 17: Cumulative frequency and boxplot of the aircraft delay times

SECTOR option disabled $\left(\mathbf{T}_{\mathbf{E}} \mathbf{S}_{\mathbf{D}}\right.$ and $\left.\mathbf{T}_{\mathbf{D}} \mathbf{S}_{\mathbf{D}}\right)$. A likely cause is the fact that most conflicts and planning problems could be resolved by a change in speed alone (which is manipulated on the TSVD) with the SECTOR option enabled. Secondly, manipulating waypoints requires a relatively high amount of clicks on the PVD. Friedman's ANOVA of the mouse click percentage showed that there was a significant effect for condition $\left(\chi^{2}(3)=30.400, p<.001\right)$. A following pair wise comparison of all conditions by the Wilcoxon test showed a significant difference of the click percentage between all conditions except between condition $\mathbf{T}_{\mathbf{E}} \mathbf{S}_{\mathbf{E}}$ and $\mathbf{T}_{\mathbf{D}} \mathbf{S}_{\mathbf{E}}$. Table 5 shows the detailed results. The significant increase in click percentage of condition $\mathbf{T}_{\mathbf{E}} \mathbf{S}_{\mathbf{D}}$ compared to condition $\mathbf{T}_{\mathbf{D}} \mathbf{S}_{\mathbf{D}}$ (both with SECTOR disabled) is likely caused by the use of the ghost view tool on the TSVD for condition $\mathbf{T}_{\mathbf{E}} \mathbf{S}_{\mathbf{D}}$.

Table 5: Pair wise comparison of the mouse click percentage per condition

\begin{tabular}{lll}
\hline \hline conditions & \multicolumn{1}{c}{$z$} & asymp. sig. (2-tailed) \\
\hline $\mathbf{T}_{\mathbf{E}} \mathbf{S}_{\mathbf{D}}<\mathbf{T}_{\mathbf{E}} \mathbf{S}_{\mathbf{E}}$ & -3.059 & $p<0.01^{* *}$ \\
$\mathbf{T}_{\mathbf{D}} \mathbf{S}_{\mathbf{E}}>\mathbf{T}_{\mathbf{E}} \mathbf{S}_{\mathbf{E}}-0.078$ & $p>0.05$ \\
$\mathbf{T}_{\mathbf{D}} \mathbf{S}_{\mathbf{D}}<\mathbf{T}_{\mathbf{E}} \mathbf{S}_{\mathbf{E}}-3.059$ & $p<0.01^{* *}$ \\
$\mathbf{T}_{\mathbf{D}} \mathbf{S}_{\mathbf{E}}>\mathbf{T}_{\mathbf{E}} \mathbf{S}_{\mathbf{D}}-3.059$ & $p<0.01^{* *}$ \\
$\mathbf{T}_{\mathbf{D}} \mathbf{S}_{\mathbf{D}}<\mathbf{T}_{\mathbf{E}} \mathbf{S}_{\mathbf{D}}-2.197$ & $p<0.05^{*}$ \\
$\mathbf{T}_{\mathbf{D}} \mathbf{S}_{\mathbf{D}}<\mathbf{T}_{\mathbf{D}} \mathbf{S}_{\mathbf{E}}-3.059$ & $p<0.01^{* *}$ \\
\hline ** significant $(p<0.01)$ & \\
$*$ significant $(p<0.05)$ &
\end{tabular}

\section{Ghost view tool}

Figure 18(b) shows a boxplot of the average time of projection when using the ghost view tool. This average time of projection is given in 'seconds in the future' with respect to the time of use. As expected, the figure shows that for all subject groups, the mean average view time lies further in the future with the SECTOR option enabled (condition $\mathbf{T}_{\mathbf{E}} \mathbf{S}_{\mathbf{E}}$ ). When the speed of the aircraft can be manipulated in the adjacent sector, the controller is able plan the traffic further ahead in time. By using a Kolmogorov-Smirnov test, the average view time was found to be normally distributed $(D(24)=0.069, p>.05)$. A repeated-measures ANOVA showed no significant effect of the average view time between the three subject groups $(F(2,9)=0.338, p=0.72)$. By testing the repeated-measures within-subject effects for all participants, the average view time was indeed found to be significantly farther in the future with the SECTOR option enabled $(F(1,9)=7.478, p<0.05)$.

\section{F. Condition questionnaire}

The most important findings of the answers to the multiple choice questions will briefly be discussed. Answers have been given on a six level Likert scale. The participants reported to have a more complete mental picture of the traffic in the conditions with the SECTOR option enabled. Secondly, the participants also reported to make more use of both 


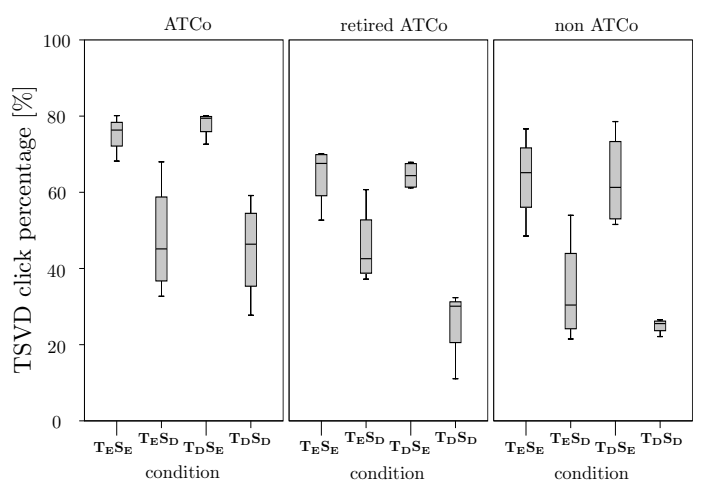

(a) TSVD mouse click percentage

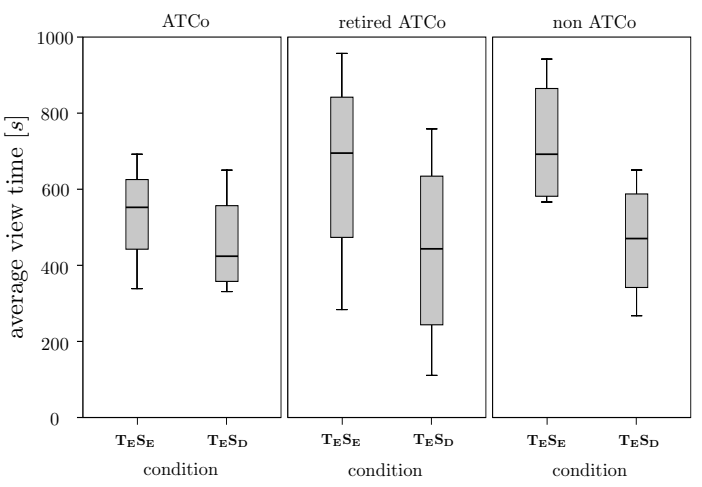

(b) Average ghost view look-forward time boxplot

Figure 18: Boxplots of the mouse click ratio and average ghost view look-forward time, per condition, clustered per subject group

screens (TSVD and PVD) in these conditions. When asked whether they thought they managed the traffic safely, all answers ranged in the top three (out of six). However, for both conditions with the TOOLS enabled, the scores were higher (always in the top two). Furthermore, the participants reported that it was clearer to them where problems would occur in the planning with the TOOLS enabled. No significant difference was found between the conditions for the answers to whether the controllers thought they could manage the traffic efficiently.

\section{G. Final questionnaire}

The most important results which followed from the final questionnaire will now be discussed. The following notation will be used to indicate participant group: non ATCo (N), retired ATCo (R), active ATCo (A). When asked about the main strength of the TSVD, seven out of twelve participants (3N, 3R, 1A) explicitly reported that this was the option to visualize the future traffic situation by means of 'ghosts'. Four other participants $(1 \mathrm{~N}, 1 \mathrm{R}, 2 \mathrm{~A})$ more generally reported that the strongest points were the tools offered by the interface (blue bars, forbidden zones, ghost view tool) to support them with making a global plan and with sequencing the traffic. Here, one ATCo also mentioned that with a good use of the interface, a high efficiency and landing capacity can be maintained, and a predictable situation can be created for the pilots. The last participant (A) reported that the strongest point was 'the planning interface'.

When asked about the main weakness of the TSVD, four out of twelve participants (2N, 1R, 1A) indicated that this was the unclear overlapping of the blue bars. Two other participants $(1 \mathrm{~N}, 1 \mathrm{~A})$ reported that this was the inability to see when aircraft were in conflict with each other (if no aircraft was selected). Three participants $(1 \mathrm{~N}, 1 \mathrm{R}, 1 \mathrm{~A})$ indicated that the weakest point was the sensitivity and the details of the 'control' of the interface. Sensitivity of dragging waypoints, $5 \mathrm{kts}$ snap to the speed profile, and double clicking to manipulate the 4DT were also mentioned. Another ATCo mentioned that the weakest point was the high mental workload, and the fact that disturbances by weather etc. were not taken into account. A retired ATCo stated that the TSVD could be much better, referring to the vertical stackview display, which is currently being developed at the LVNL. Furthermore, an ATCo commented that the weakest point was that one could lose themselves in time; when controlling the traffic 20 minutes ahead in time, one could easily forget the present situation.

Several questions were presented about the influence of various aspects to the level of difficulty of the experiment. Ten out of twelve participants indicated that the limited time to learn with the TSVD did not have a significant contribution to the overall difficulty, indicating that the 45 minute briefing was sufficient to learn the basics of the interface. All participants agreed that the limited mix of traffic (no transit and outbound aircraft) made the level of the test easy or reasonably easy. Furthermore, both the answers concerning the difficulty due to the amount of controlled aircraft and due to the way in which the traffic was delivered to the sector varied strongly between the subjects. Additionally, inexperience as an ATCo $(\mathrm{N})$, bunching in some scenarios $(\mathrm{N})$ and inexperience of working with PC-based tools $(\mathrm{R})$ were mentioned as factors which contributed to a higher level of difficulty. 


\section{Manipulation of the $4 D T$}

Questions were presented whether the participants liked to use the three methods to manipulate the 4DT which were supported by the interface. The answers had to be rated on a 4 level Likert scale: agree, partially agree, partially disagree, and disagree. Figure 19 shows the results. Note that half points were used when a participant marked two answers. The figures show that almost all participants liked the option to manipulate the arrival time and horizontal route. On the other hand, manipulating the vertical profile was less preferred. This is probably caused by the fact that only five participants used to option to manipulate the vertical profile during the experiment (for a total of nine aircraft). Additional comments were also given: one participant $(\mathrm{N})$ stated that route control provides many options to control the traffic. One ATCo commented that a 'holding' function in case of extensive delays would be a welcome addition. Two participants $(1 \mathrm{~N}, 1 \mathrm{R})$ added an extra comment that they made little use of the option to manipulate the vertical profile, and one participant $(\mathrm{N})$ commented that this option is only useful when the trajectories of aircraft cross each other en-route. When asked about the intuitiveness of the control options, the ratings concerning arrival time and route were similar to the previous question. However, the intuitiveness of manipulating the vertical profile leaned more towards the positive: agree $(2 \mathrm{~N}, 1 \mathrm{R}, 1 \mathrm{~A})$, partially agree $(2 \mathrm{~N}, 1 \mathrm{R}, 1 \mathrm{~A})$, partially disagree $(2 \mathrm{R}, 1 \mathrm{~A})$, and disagree (1A).

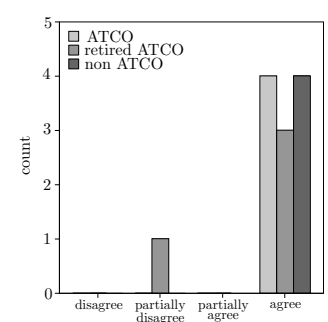

(a) Arrival time

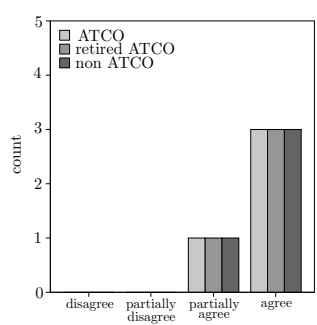

(b) Horizontal route

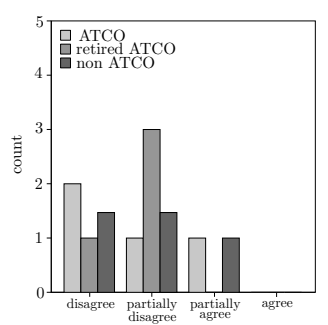

(c) Vertical profile

Figure 19: Answers concerning the manipulation of the 4DT. I like to use the option to manipulate the ...

\section{Ghost view tool and conflict visualization on the PVD}

The participants were asked whether the conflict visualization on the PVD and the ghost view tool increased their insight into the traffic situation. Secondly, the participants were asked whether the mapped (across-screen) information was intuitive to interpret. Figure 20 shows the results. The figure shows that all participants agree or partially agree that the ghost view tool increases their insight into the traffic and works intuitively. However, the opinions concerning conflict visualization on the PVD are more divided. An additional comment was made $(\mathrm{N})$ that the ghost view tool takes some time to get used to, but afterwards works intuitively. Secondly, it was commented (N) that the conflict detection on the PVD only shows that something is wrong along the trajectory, but not exactly how the conflict can be resolved (horizontal, vertical or speed).

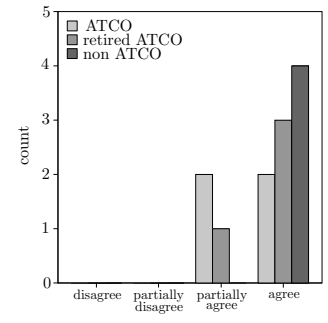

(a) Ghost view insight

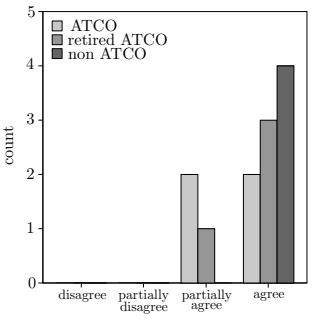

(b) Ghost view intuitive

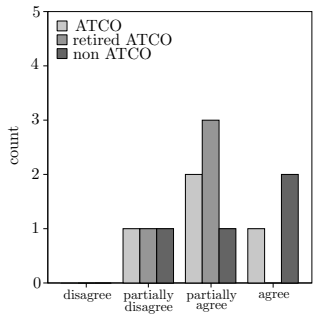

(c) Conflict insight

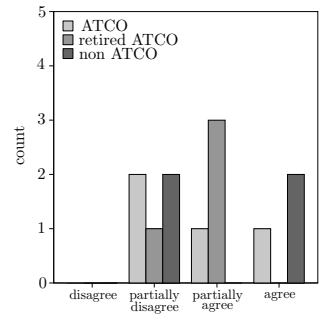

(d) Conflict intuitive

Figure 20: Answers concerning the ghost view tool and conflict visualization on the PVD 


\section{Discussion}

The results validate that the TSVD can be used alongside the conventional PVD to support the ACC-ATCo with planning and managing inbound traffic in a future trajectory-based form of ATC. This is underlined by the fact that all participants were able to guide the traffic into the TMA safely (conflict free) without extensive training.

Furthermore, no significant difference was noticeable in the overall performance between the three subject groups. The participants who have never actively controlled aircraft performed at the same level as the active ATCos. This can be attributed to the fact that the trajectory-based form of ATC is very different to the current form of ATC, and is therefore a new way of working for all participants. In addition, the fact that outbound and transit aircraft were not included simplified the experiment, and only provided a limited representation of the current ATC work domain.

The situation awareness and workload scores have been shown to be significantly better when the SECTOR option was enabled. A number of causes could have contributed to this. Firstly, it has been shown that the paths of less aircraft had to be stretched inside the own sector. As hypothesized, the increase in situation awareness could be explained by the fact that most aircraft flew in a straight line from the sector border to the fix, creating a more predictable and easily interpretable flow of traffic. Workload is then also reduced because changes to speed require fewer actions by the controller than manipulating the horizontal route. Secondly, when the SECTOR option is enabled, the controller has a more complete picture of the traffic on the TSVD. This makes it easier to see where sequencing and separation problems will occur in the future (overlapping blue bars and forbidden zones). Because the controller is able to manipulate the speed of all aircraft, most identified problems can immediately be resolved. In addition, aircraft which are further away have a larger margin in arrival times, thus more options for sequencing, and do not require immediate attention. As a consequence, the temporal demand of planning the traffic could be reduced as the separation and sequence can be determined long before it becomes an acute problem.

This increase in SA and decrease in workload, even with the higher amount of controllable aircraft, can also be linked to the 'first come, first serve' planning strategy which participants frequently reported to use. With this method the sequence is planned in a pair wise manner; i.e., fix the 4DT of the closer aircraft, plan the following (later) aircraft behind it. Consequently, the controllers' attention moves from aircraft which require the most immediate attention (closest to the fix and smallest margin of arrival times) to the aircraft which require the least.

Furthermore, the aircraft count itself becomes less important because all trajectories are fixed. Once the traffic is managed, little attention has to be paid to the monitoring task. This could be an indication that the size of the sector could be increased for the trajectory-based form of ATC, without a significant reduction of SA or increase in workload.

A trend towards a higher level of situation awareness has been found with the TOOLS enabled. However, these results showed no significance. A reason for this could be that situation awareness scores were already relatively high for all conditions. Also, using the ghost view tool requires extra actions by the controller (moving the attention away from the actual planning task), and therefore could explain the non-significant reduction of workload. The fact that most participants reported the ghost view tool to be the strongest element of the TSVD does however validate its usefulness for creating an insight in the dynamic traffic flow.

The opinions about the conflict visualization on the PVD varied strongly between the participants. As one participant stated in the final questionnaire; the spatial location along the trajectory which is in conflict with other traffic is shown, but unlike the forbidden zones in the TSD or VSD, it does not provide a direct cue how to resolve the conflict. This could be improved by graphically indicating 'forbidden areas' on the PVD in which the paths of aircraft cannot be stretched (such as in the Phare Ground Human Machine Interface (GHMI) project ${ }^{15}$ ).

One of the main recommendations which followed from the previous research was to incorporate the vertical plane in the interface to make it more complete. Many participants, however, indicated that they did not use the information on the VSD, and did not make use of the vertical plane to separate aircraft at all. This was confirmed by the fact that the vertical profile of only nine out of 816 controlled aircraft had been manipulated during the experiment. A cause could be that the initial traffic situations of the scenarios were built up such that there were no crossing aircraft. Maintaining separation was mainly assured by changing the route or speed to merge the aircraft at the fix. Secondly, the size of the controlled sector was relatively small. The options to manipulate the vertical profile were limited because many aircraft already initiated their descent close to the sector border. When the size of the sector is increased and outbound and transit aircraft are added to the simulation, the vertical profile is likely to become a more important control variable. 


\section{Conclusion and recommendations}

Experimental results validate that the TSVD can support an ATCo with managing inbound traffic safely and efficiently in a future trajectory-based form of ATC. No loss of separation has been recorded. The addition of two new tools to the interface showed a trend towards better situation awareness. However, these results were not significant. The option to dynamically project the time-evolution of the planned future traffic situation on the PVD (ghost view tool) was reported to be the strongest point of the TSVD. The importance of multi-sector coordination and the size of the controlled sector has been underlined by the significant increase of situation awareness and decrease of controller workload, which has been measured for conditions in which speed changes could already be issued to aircraft in the adjacent sector.

For further development of the TSVD, it is recommended to investigate the form in which outbound and transit aircraft could be incorporated to make it more complete. The TMA planning (blue bars) should also be improved, making it more apparent where merge problems will occur. Furthermore, when no aircraft is selected, cues should be given for the existence and the priority of conflicts. When manipulating the horizontal path of an aircraft, forbidden areas could be indicated on the PVD in which it is unsafe to reroute. Dealing with disturbances such as wind, bad weather, inaccuracies in trajectory prediction and other unforeseen events have not yet been investigated. The size of the controlled sector has been shown to have significant influence on controller workload, situation awareness and to the efficiency of the traffic flow, but was not taken as an explicit experiment variable. Therefore, further research is required to determine this relationship. Finally, the TSVD is based upon a mature state of four-dimensional operations in which all aircraft have full 4D capabilities. In order to gain operational acceptance, solutions should be found for a transition period in which both the air- and ground segment are only partially equipped.

\section{References}

${ }^{1}$ Oprins, E. A. P. B. and Schuver, M., "Competentiegericht opleiden en beoordelen bij LVNL [Competence based training and assessment at LVNL]," HUFAG nieuwsbrief, , No. 6, 2003, pp. 2-4.

${ }^{2}$ Oprins, E. A. P. B., Design of a competence-based assessment system for air traffic control training, Ph.D. dissertation, Faculty of Economics and Business Administration, Maastricht University, Maastricht, The Netherlands, 2008.

${ }^{3}$ Dlugi, O., Astheimer, T., Baldoni, C., Bara, J. M., Brown, R., Cato, R., O’Connell, G., Standar, M., Stenson, J., and Tauss, G., "The ATM Target Concept," Deliverable 3 DLM-0612-001-02-00, SESAR Consortium, Toulouse, France, 2007.

${ }^{4}$ Dlugi, O., Astheimer, T., Baldoni, C., Brown, R., Cato, R., Erb, M., Hilton, D., O'Connell, G., Sohn, A., and Stenson, J., "The ATM Deployment Sequence," Deliverable 4 DLM-0706-001-02-00, SESAR Consortium, Toulouse, France, 2008.

5 "Concept of Operations for the Next Generation Air Transportation System. Version 2.0." Report, Joint Planning and Development Office, 2007.

${ }^{6}$ Slattery, R. A., "Examination of Possible Future Terminal Area Procedures," AIAA Guidance, Navigation, and Control Conference, San Diego, California, July 29-31 1996.

${ }^{7}$ Ballin, M. G., Hoekstra, J. M., Wing, D. J., and Lohr, G. W., "NASA Langley and NLR Research of Distributed Air/Ground Traffic Management," AIAA's Aircraft Technology, Integration and Operations 2002 Technical, Los Angeles, California, USA, October 1-3 2002.

${ }^{8}$ Green, S. M., Bilimoria, K. D., and Ballin, M. G., "Distributed Air-Ground Traffic Management for En Route Flight Operations," AIAA Guidance, Navigation, and Control Conference and Exhibit, Denver, Colorado, August 14-17 2000.

${ }^{9}$ Prevot, T., Lee, P. U., Smith, N., and Palmer, E. A., "ATC Technologies for Controller-Managed and Autonomous Flight Operations," AIAA Guidance, Navigation and Control Conference, San Francisco, California, USA, August 15-18 2005.

${ }^{10}$ Celio, J. C., "Performance-Based ATM: Concept and Service Provider Roles and Responsibilities," AIAA Aviation Technology, Integration and Operations Conference, Belfast, Northern Ireland, September 18-20 2007.

${ }^{11}$ Wichman, K. D., Lindberg, L. G. V., Kilchert, L., and Bleeker, O. F., "Four-Dimensional Trajectory Based Air Traffic Management," AIAA Guidance, Navigation and Control Conference, Providence, Rhode Island, USA, August 16-19 2004.

${ }^{12}$ Van Dijk, E., Mulder, M., Van Paassen, M. M., and Roerdink, M. I., "An Interface for Inbound Traffic Management by Air Traffic Control," Proceedings of the AIAA Guidance, Navigation and Control Conference, Chicago (IL), USA, August 10-13, , No. AIAA 2009-6164, 2009.

${ }^{13}$ Endsley, M. R., "Situation Awareness, Automation \& Free Flight," Presented at the FAA/Eurocontrol Air Traffic Management R\&D Seminar, Saclay, France, June 1997.

${ }^{14}$ Whiteley, M. J. and Wilson, I., "PHARE Advanced Tools Problem Solver," Final Report PHARE/EEC/PAT-6.5/FR; 1.0, DOC 98-70-18, Eurocontrol, Brussels, Belgium, August 1999.

${ }^{15}$ Jorna, P. G. A. M., Pavet, D., Van Blanken, M., and Pichancourt, I., "PHARE Ground Human Machine Interface (GHMI) project," Summary Report PHARE/NLR/GHMI-7/FR/1.0, DOC 99-70-02, Eurocontrol, Brussels, Belgium, August 1999.

${ }^{16}$ Heesbeen, W. W. M., Hoekstra, J. M., and Valenti Clari, M. S. V., "Traffic Manager, Traffic Simulation for Validation of Future ATM Concepts," AIAA Modeling and Simulation Technologies Conference and Exhibit, Austin, Texas, USA, August 11-14 2003.

${ }^{17}$ Vicente, K. J. and Rasmussen, J., "Ecological Interface Design: Theoretical Foundations," IEEE Transactions on Systems, Man, and Cybernetics, Vol. 22, No. 4, July/August 1992, pp. 589-606.

${ }^{18}$ Wubben, F. J. M. and Busink, J. J., "Environmental benefits of continuous descent approaches at Schiphol Airport compared with conventional approach procedures," Report NLR-TP-2000-275, National Aerospace Laboratory NLR, division Air Transport, Amsterdam, The Netherlands, May 2000. 
${ }^{19}$ Meijer, L. K., De Gelder, N., Mulder, M., Van Paassen, M. M., and In 't Veld, A. C., "Time-based Spaced Continuous Descent Approaches in Busy Terminal Manoeuvring Areas," Proceedings of the AIAA Guidance, Navigation and Control Conference, Chicago (IL), USA, August 10-13, , No. AIAA 2009-6165, 2009.

${ }^{20}$ Endsley, M. R., Theoretical Underpinnings of Situation Awareness: A Critical Review. In Eds M. R. Endsley and D.J.Garland, Situation Awareness and Measurement (Ch.1), Lawrence Erlbaum Associates, Mahwah, NJ, 2000.

${ }^{21}$ Hutchins, E. L., Hollan, J. D., and Norman, D. A., "Direct Manipulation Interfaces," Human-Computer Interaction, Vol. 1, 1985, pp. 311338.

${ }^{22}$ Woods, D. D., "Visual Momentum: A concept to improve the cognitive coupling of person and computer," International Journal of ManMachine Studies, Vol. 21, No. 3, September 1984, pp. 229-244.

${ }^{23}$ Unknown, "Standard Aircraft Modeling Interface," Users guide (draft) rev. 4.2, Eurocontrol Headquarters, Brussels, Belgium, April 2009.

${ }^{24}$ Unknown, "G.A.M.E. Aircraft Performance Model," Report ver. 2.0, Eurocontrol Headquarters, Brussels, Belgium, September 2002.

${ }^{25}$ Hart, S. G. and Staveland, L. E., "Development of NASA-TLX (Task Load Index): Results of empirical and theoretical research," Human Mental Workload, edited by P. A. Hancock and N. Meshkati, Elsevier Science Publishers (North-Holland), Amsterdam, 1988, pp. $139-183$.

${ }^{26}$ Straeter, O., Woldring, V. S. M., Barbarino, M., Skoniezki, A., and Philipp, W., "The Development of Situation Awareness Measures in ATM Systems,” Tech. Rep. HRS/HSP-005-REP-01; 1.0, Eurocontrol Headquarters, Brussels, Belgium, June 2003. 